\title{
Catastrophic Disruptions Revisited
}

\author{
Willy Benz \\ Physikalisches Institut \\ University of Bern \\ Switzerland \\ email: wbenz@phim.unibe.ch \\ and \\ Erik Asphaug \\ Earth Sciences Department \\ University of California \\ Santa Cruz, CA 95064 \\ email: asphaug@cosmic.ucsc.edu
}

To appear in Icarus

\begin{abstract}
We use a smooth particle hydrodynamics method (SPH) to simulate colliding rocky and icy bodies from cm-scale to hundreds of $\mathrm{km}$ in diameter, in an effort to define self-consistently the threshold for catastrophic disruption. Unlike previous efforts, this analysis incorporates the combined effects of material strength (using a brittle fragmentation model) and self-gravitation, thereby providing results in the "strength regime" and the "gravity regime", and in between. In each case, the structural properties of the largest remnant are examined.

Our main result is that gravity plays a dominant role in determining the outcome of collisions even involving relatively small targets. In the size range considered here, the enhanced role of gravity is not due to fracture prevention by gravitational compression, but rather to the difficulty of the fragments to escape their mutual gravitational attraction. Owing to the low efficiency of momentum transfer in col-
\end{abstract}


lisions, the velocity of larger fragments tends to be small, and more energetic collisions are needed to disperse them.

We find that the weakest bodies in the solar system, as far as impact disruption is concerned, are about $300 \mathrm{~m}$ in diameter. Beyond this size, objects become more difficult to disperse even though they are still easily shattered. Thus, larger remnants of collisions involving targets larger than about $1 \mathrm{~km}$ in radius should essentially be selfgravitating aggregates of smaller fragments.

\section{Introduction}

Solar system evolution hinges on the outcome of collisions. In order for planetary accretion to proceed, for example, collisions must result in larger, not smaller pieces. Asteroid dynamical families are in most cases thought to be the outcome of catastrophically disruptive collisions between parent bodies; smaller asteroids and interplanetary dust may be the result of collisional cascades; asteroid and planetary binaries (Ida and Dactyl, Pluto and Charon, Earth and Moon) may also be an expression of impact.

Impacts or collisions can be grouped in three different categories depending on outcome: cratering, shattering and dispersing. The first category is defined by events leading to the formation of topographical signatures (craters) accompanied by ejection of material but without affecting the physical integrity of the main body. Shattering impacts, on the other hand, are events that break the parent body into smaller pieces. Dispersing events are those which not only break the body into pieces, but also manage to impart velocities to those fragments in excess of escape velocity. Much observational evidence testifies to these most energetic events: the dynamical asteroid families for example (such as Koronis), and the iron meteorites which are fragments excavated by impact from the cores of differentiated bodies.

It has become customary in the literature to characterize impacts in terms of a specific energy threshold (the kinetic energy in the collision divided by target mass). The threshold for a shattering event is defined by $Q_{S}^{*}$, the specific energy required to break a body into a spectrum of intact fragments, the largest one having exactly half the mass of the original target. Dispersing events, on the other hand, are defined by $Q_{D}^{*}$, the specific energy required to disperse the targets into a spectrum of individual but possibly reaccumulated objects, the largest one having exactly half the mass of the original target. 
In the strength regime, where gravity does not matter (fragments do not reaccumulate), $Q_{S}^{*}$ is obviously equal to $Q_{D}^{*}$. In the gravity regime however, $Q_{D}^{*}$ is always greater than $Q_{S}^{*}$, since the target must be fragmented and also dispersed by the event.

Laboratory experiments can be designed to determine this threshold for small targets, i.e. targets in the strength dominated regime (see for example Fujiwara et al. 1989; Davis \& Ryan 1990; Ryan et al. 1991). By using up to meter-sized targets, Housen and Holsapple (1999) were able to confirm previous theoretical prediction of strength weakening with size. Impacts in pressurized targets (Housen 1991) designed to simulate self-gravitating bodies indicate that strength increases again in these "larger" targets. However, these artificially pressurized targets are uniformly compressed while in truly self-gravitating bodies the overburden is a function of position. The interpretation of these experiments is therefore not completely straightforward.

The scales of planetary impacts are far different from what can be studied directly in the laboratory, and extrapolations by more than a dozen orders of magnitude in mass are required before reaching a range relevant to asteroids and/or planetesimals. Detailed theoretical models of disruptive impacts (Holsapple \& Housen 1986; Housen \& Holsapple 1990; Holsapple 1994) try to bridge this gap by establishing relations among non-dimensional ratios involving impactor size, impact velocity, target strength, density, etc. Such relations, deriving from dimensional analysis, assume uniformity of process, structural continuity and other idealizations, and cannot predict detailed outcomes.

Recent exponential increases in computational power have enabled numerical simulations to become the method of choice to investigate these issues in greater detail. Laboratory impact experiments are used to validate the numerical models on small scales before extrapolations to sizes relevant to solar system bodies are undertaken. However, numerical attempts to determine $Q_{D}^{*}$ have for the moment been limited to idealized geometry (twodimensional axisymmetry) (Ryan \& Melosh 1998) or restricted to a subset of target sizes (gravity regime) (Melosh \& Ryan 1997; Love \& Ahrens 1996) and low resolution (Love \& Ahrens 1996).

In this paper, we use a smooth particle hydrodynamics method (SPH) to simulate colliding basalt and icy bodies from $\mathrm{cm}$-scale to hundreds of $\mathrm{km}$ in diameter, in an effort to define self-consistently the threshold for catastrophic disruption. Unlike previous efforts, this analysis incorporates the combined effects of material strength (using a brittle fragmentation model) 
and self-gravitation, thereby providing results in the "strength regime" and the "gravity regime" and in between. We begin with a short presentation of the physical model (section 2) followed by a discussion of various numerical issues pertaining to our study (section 3). The results obtained from 480 different simulations are presented and discussed in section 1 .

\section{Physical Model}

Our approach of dynamical fracture modeling has been described in detail elsewhere (Benz and Asphaug 1994, 1995) and will only be summarized here.

The equations describing an elastic solid are the usual conservation equations (mass, momentum and energy) in which the stress tensor has a nondiagonal part, the so-called deviatoric stress tensor. With the assumption that the stress deviator rate is proportional to the strain rate, i.e. Hooke's law, and a suitable equation of state (see section 2.1) it is possible to numerically solve this set of coupled differential equations. Plasticity is introduced by suitably modifying the stresses beyond the elastic limit using a von Mises yielding relation. Not counting the equation of state, this approach requires 3 material-dependent constants: $\mu$ the shear modulus, $Y$ elastic limit and $E_{\text {melt }}$ the melt energy used to decrease the elastic limit with increasing temperature. Young's modulus can be computed from the knowledge of the bulk modulus (parameter $A$ in section 2.1) and the shear modulus according to $E=9 A \mu /(3 A+\mu)$.

For the lower tensile stresses associated with brittle failure, we use a fracture model based on the nucleation of incipient flaws whose number density is given by a Weibull distribution (Weibull 1939; Jaeger \& Cook 1969)

$$
n(\epsilon)=k \epsilon^{m}
$$

where $n$ is the number density of flaws having failure strains lower than $\epsilon$. The Weibull parameters $m$ and $k$ are material constants which can be determined from laboratory experiments comparing failure stress to strain rate, although data are scarce. Table 1 gives the numerical values of these material-dependent constants used in this study. The Weibull parameters for basalt are a previously-determined (Benz \& Asphaug 1995) best match to the laboratory impact experiments of Nakamura \& Fujiwara (1991); parameters for ice are derived by fitting a line to the measurements of rate-dependent fracture stress published in Lange \& Ahrens (1983). 
Once flaws are activated, fractures grow at constant velocity, about half the sound speed. The extent to which fracture affects the local properties of matter is described by a scalar state variable called damage. The possible values for $D$ range between 0 (undamaged) to 1 (totally damaged). The ability to sustain shear or tension by individual particles is reduced linearly with $D$ and vanishes for $D=1$. Because damage accrues according to the entire stress history of a parcel of matter, only Lagrangian solutions to the hydrodynamics equations are applicable.

Our model of dynamic fragmentation explicitly reproduces the growth of cracks in a brittle elastic solid by rupturing bonds and forming new free surfaces. Cracks grow when local failure strains are exceeded, and stresses are relieved across the crack boundaries. The release of stress along fracture walls increases differential stress at the crack tips, driving cracks forward in the manner of an actual brittle solid. Tensile and shear stresses are unsupported across disconnected regions, leading to reduced average strength and sound speed (i.e. damage) in the body. By producing actual cracks and fragments, our method at sufficiently high resolution automatically takes into account friction between fragments and bulking, effects which are included as recipes in statistical damage models.

\subsection{Equation of State}

We used the so-called Tillotson equation of state (Tillotson 1962) which was specifically derived for high-velocity impact computations. This equation has the advantage of being computationally expedient while sophisticated enough to allow its application over a wide regime of physical conditions.

For compressed regions and for cold expanded states where the energy density $(E)$ is less than the energy of incipient vaporization $\left(E_{\mathrm{iv}}\right)$ the Tillotson eos has the form:

$$
P=\left[a+\frac{b}{\left(E /\left(E_{0} \eta^{2}\right)+1\right)}\right] \rho E+A \mu+B \mu^{2}
$$

where $\eta=\rho / \rho_{0}$ and $\mu=\eta-1$, such that $\rho$ is the compressed density and $\rho_{0}$ is the zero-pressure density. Here $a, b, A, B$, and $E_{0}$ are referred to as the material-dependent Tillotson parameters.

For expanded states, when the internal energy $(E)$ is greater than the energy of complete vaporization $\left(E_{\mathrm{cv}}\right)$, the pressure has the form 


$$
P=a \rho E+\left[\frac{b \rho E}{\left(E /\left(E_{0} \eta^{2}\right)+1\right)}+A \mu e^{-\beta\left(\rho_{0} / \rho-1\right)}\right] e^{-\alpha\left(\rho_{0} / \rho-1\right)^{2}}
$$

where $\alpha$ and $\beta$ are constants that control the convergence rate of the equation to the perfect gas law. For intermediate states, pressure is simply interpolated linearly between both expanded and compressed states.

Tillotson parameters for a variety of geologic materials have been compiled by Melosh (1989). However, the most fundamental coefficients (especially density and bulk modulus) for candidate materials are typically different from those reported for specimens used in laboratory impact fragmentation experiments, from which our Weibull coefficients (Table 1) are derived. We therefore make the following alteration to the equations of state, in an effort to best characterize the material in the fragmentation experiments: We substitute the measured density for the published Tillotson reference density of the most similar material, and the measured bulk modulus for the Tillotson parameter $A$. Because the Tillotson parameter $B$ is not known for the specific target material, we make use of the fact that $B \simeq A$ for most geologic materials. This is probably a good assumption for basalt but may underestimate the second-order pressure terms in ice, making it more "rock-like". We summarize in Table 8 the Tillotson parameters used in this paper. This situation is not ideal. However, given the rudimentary understanding of asteroidal and cometary compositions (they are surely neither pure basalt nor pure water ice!), we feel that there is little to be gained until comprehensie experimental work, combining dynamic strength and equation of state, can be performed on more representative candidate materials at the appropriate temperature.

\section{$3 \quad$ Numerical Issues}

In this section we discuss various issues related to the numerical methods used to either simulate the impacts or analyze the results of the simulations.

\subsection{Impact Simulations}

Fracture depends on the entire stress history of a given piece of material. A Lagrangian approach, in which the frame of reference is attached to the material, is therefore the natural framework for solving the equations briefly 
described in section 2; Eulerian codes to date cannot accurately follow stress history and the development of cracks. Conventional Lagrangian codes, however, are unable to handle large material deformations, as tangling and deformation of the grid severely affect the accuracy of derivatives.

Smooth Particle Hydrodynamics (see reviews by Benz 1990; Monaghan 1992) does not suffer from this problem. We have developed a three-dimensional SPH code capable of simulating dynamical fracture of brittle material (Benz \& Asphaug 1994, 1995). Our SPH code being an explicit code, the size of the time step is limited by the Courant condition. In practice, this means that the time step cannot exceed a fraction of the time needed by an elastic wave to cross a resolution element. If this element is of size $h$, numerical stability requires that $d t<h / c$, where $c$ is the wave speed. With 42,000 particles, $h$ is of order $R / 35$ where $R$ is the target radius. As an estimate for wave speed we take $c=\sqrt{A / \rho}$ where $A$ is the bulk modulus. Taking the values appropriate for basalt, we get $c=3.2 \mathrm{~km} / \mathrm{s}$ which leads to an upper bound for the time step given by $d t<9 \times 10^{-3} R$, where $d t$ is in seconds and $R$ in $\mathrm{km}$.

Gravitational clumping of fragments and the formation of rubble piles on the other hand takes place on a dynamical timescale given by $\tau=\sqrt{3 \pi / 16 G \rho}$. For basalt, we obtain a value for the clumping timescale of $\tau=1.8 \times 10^{3} \mathrm{~s}$. The number of timesteps required to follow a collision up to the time where gravitational reaccumulation occurs is therefore $N>\tau / d t=2 \times 10^{10} / R$. For targets of radius $100 \mathrm{~m}$, this requires a number of timesteps greater than $10^{6}$, which is currently prohibitive.

While these two vaslty different timescales prevent us from simulating the entire process directly, they also mean that self-gravitation is entirely decoupled from the impact phase for intermediate targets (see Asphaug 1997). Clumping can therefore be studied independently as part of the postprocessing analysis of the impact simulations proper (see section 3.3). This has also the additional advantage not having to solve Poisson's equation (self-gravity) during the impact phase. We therefore use a faster linked list in rank space algorithm to find the neighbor particles rather than a hierarchical tree. While the dynamical effects of self-gravity are not included in the simulations, the fracture shielding effect due to compression is included in the code(see section 3.2).

The code's behavior in the elastic regime was extensively tested against simple analytical solutions, while the fracture modeling was tested by simu- 
lating laboratory impact experiments (Nakamura and Fujiwara 1991; Ahrens and Rubin 1993) leading to core-type and radial fragmentation and extensive spallation. The code was able to reproduce the laboratory experiments to a level of detailed accuracy never achieved before, including shape, ejection speed and rotation of fragments, trajectories of far-field fracture, and post-fragmentation sound speed relationships.

\subsection{Flaw Assignments}

We use the explicit flaw assignment procedure described in Benz \& Asphaug (1995) using the material-dependent Weibull parameters listed in Table 1. Our explicit flaw method improves upon the mixed explicit-implicit method of Benz \& Asphaug (1994). These methods are wholly independent of numerical resolution (in terms of the assigned flaw statistics) and lead to rateand size-dependent fracture thresholds.

Each particle is assigned a number of discrete fracture thresholds distributed at random from the underlying Weibull distribution (Eq. 1). When local stresses exceed such a threshold, damage $D$ is allowed to grow until the local stresses decrease again, or until $D$ reaches its maximum authorized value. This maximum authorized value for particle $i$ is given by $D_{\max }^{i}=N_{a}^{i} / N_{\max }^{i}$ where $N_{a}^{i}$ is the currently active number of flaws and $N_{\max }^{i}$ the number of fracture thresholds assigned to particle $i$. This particular algorithm of damage accrual and authorization ensures that flaw distribution and activation are not linked to numerical resolution.

For fracture to proceed, stresses must first overcome the local gravitational overburden. To include what amounts to an effective gravitational strengthening without having to solve the full Poisson equation during the simulations, we use the technique (Asphaug and Melosh 1993) of adding to the local stress tensor (determined from solving the elasto-dynamical equations) an isotropic lithostatically equilibrated stress $\left(P_{l}(r)=\frac{2 \pi}{3} G \rho^{2}\left(R^{2}-r^{2}\right)\right)$. The sum, the "total fracture stress", is converted into a Weibull strain for use in Eq. 1 by dividing the largest tensile principal stress by the modified Young's modulus, as pioneered by Melosh et al. (1992). Note that the difference between the rock fracture stress and the total fracture stress is negligible for targets smaller than a few 10's of km radius, and that for larger targets, strengthening towards the interior may be due as much to thermodynamical processes (annealing) than to gravity per se. 


\subsection{Fragment search}

As outlined above, late time gravitational evolution can for intermediate size targets be decoupled from the impact physics itself. Thus, we perform the characterization of the collisional outcome as a postprocessing step. Our approach to find the final collisional outcome proceeds in two steps. First, and regardless of target size, we begin by searching for intact (undamaged) fragments, i.e. fragments that are held together by material strength alone. Our method makes no assumptions regarding their number, geometry, or location, as they are a natural outcome of the fracture trajectories resulting from a given simulation. Contrary to statistical fragment approaches, our fragments are explicitly defined by the network of cracks resulting from the impact. We use a friends-of-friends algorithm to identify individual monolithic fragments defined as contiguous regions of particles held together by strength and surrounded by strengthless or empty regions. At the end of this procedure, we obtain for each fragment its mass, position, velocity, angular momentum and moment of inertia. This is the "shattering" spectrum of fragment sizes discussed in the Introduction.

As soon as the target size exceeds 50-100m, searching for intact fragments is no longer sufficient, as some of them may be able to reaccumulate due to gravity, leading to remnants incorporating multiple fragments. We search for these gravitationally-bound aggregates by applying a well known iterative procedure adapted from the techniques used in simulations of galaxy formation. The procedure starts by computing the binding energy of all particles and/or fragments with respect to the largest fragment, or if too small, the particle closest to the potential minimum. This serves as the seed for nucleating the total bound mass. Unbound particles are discarded, and the center of mass position and velocity of the aggregate is computed. The binding energy of all the remaining fragments and particles with respect to this new position and velocity is again computed. Unbound particles are again discarded and the procedure iterated until no particles are discarded. Typically, convergence is achieved after a few iterations $(\approx 5-10)$ with only very few particles being lost after the first $2-3$ steps. Finally, we check that particle/fragment members of this gravitationally bound aggregate are also close spatially, using again a friends-of-friends algorithm. Mass, position, velocity, angular momentum and moment of inertia are also determined for this gravitationally bound aggregate, which can be made of a collection of smaller fragments and/or individual particles. Because of the limited number 
of particles used, we limit our search to the single largest aggregate and do not attempt to search for smaller ones.

The algorithm has been tested extensively in both the strength and gravity dominated regime by comparing end results of simulations with predictions made at early times by the cluster finding algorithm. To overcome the time step problem in the gravity dominated regime, purely hydrodynamical simulations were used to allow the code to compute to sufficiently late times.

For each simulation, we therefore identify and characterize the largest object present at the end of the collision. This object may either be an intact fragment (in the strength dominated regime) or a gravitationally bound aggregate of fragments (gravity dominated regime). In the latter case, we also search for the largest intact fragment belonging to the aggregate (see Fig. (1), e.g. the largest component of the resulting "rubble-pile".

\subsection{Initial Conditions}

We have considered 8 different target radii: $3 \mathrm{~cm}, 3 \mathrm{~m}, 300 \mathrm{~m}, 1 \mathrm{~km}, 3 \mathrm{~km}, 10 \mathrm{~km}$, $30 \mathrm{~km}$, and $100 \mathrm{~km}$, and two different material types: basalt and ice. For each target we have computed impacts for five angles of incidence measured from the surface normal: $0,30,45,60$, and 75 degrees. For each material we have considered two impact velocities: for icy targets $0.5 \mathrm{~km} / \mathrm{s}$ and $3 \mathrm{~km} / \mathrm{s}$; for basalt targets $3 \mathrm{~km} / \mathrm{s}$ and $5 \mathrm{~km} / \mathrm{s}$. In each case, $Q_{D}^{*}$ was computed from a parabolic fit to three different simulations in which only the impactor mass was modified. The entire study therefore represents a total of 480 different simulations which were automatically handled by special "driver" software. As each simulation can take up to a few days of high performance workstation time, this represents a significant body of computational effort.

Because of the statistical nature of this study, we had to limit ourselves to a relatively small number of particles. In all cases, we used 42,000 particles to model the target. This number was found in convergence-test comparisons between numerical simulations and laboratory impact experiments (Benz \& Asphaug 1994, 1995) to be sufficient to determine reliably the characteristics (size, velocity and rotation) of the largest fragment. Thus in all what follows, we shall concentrate only on the characteristics of the largest fragment.

The projectile was modeled using 800 particles for the $3 \mathrm{~km} / \mathrm{s}$ and $5 \mathrm{~km} / \mathrm{s}$ impacts and 7,000 for $0.5 \mathrm{~km} / \mathrm{s}$ impacts since in this case the impactor was much larger. (In fact, in the case of $100 \mathrm{~km}$ large icy targets and large incidence angles the required projectile for a given value of $Q$ was sometimes 
bigger than the target!) The simulations were carried out in time until no further significant changes occurred in the extent of damage endured by the target and in the velocity of the ejected particles.

\section{Results}

\subsection{Catastrophic disruption threshold $Q_{D}^{*}$}

For a given impact geometry, velocity and target (parent body) material, $Q_{D}^{*}$ is determined by interpolation between three different simulations spanning a range of incident kinetic energy per unit target mass $(Q)$ chosen to yield largest remnant masses $\left(f_{\max }=M_{\mathrm{lr}} / M_{\mathrm{pb}}\right)$ generally in the range $0.3-0.7$. In the expression above, $M_{\mathrm{lr}}$ represents the mass of the largest remnant (including gravitational reaccumulation if applicable) and $M_{\mathrm{pb}}$ the mass of the parent body (or target). A parabolic fit $\left(f_{\max }=a Q^{2}+b Q+c\right)$ to these results is computed and $Q_{D}^{*}$ determined by solving for $f_{\max }\left(Q_{D}^{*}\right)=0.5$. The results of these calculations are shown in Figs. 2, 3, 1 and 5 for the various velocities and material type. In these figures, each dot represents the value of $Q_{D}^{*}$ determined from the parabolic fit. The dots corresponding to the same angle of incidence of the projectile are connected by a solid line.

We recover in these figures the well known functional dependency of $Q_{D}^{*}$ with target size, namely that $Q_{D}^{*}$ decreases with size for small targets while it increases with size for larger targets. These two behaviors correspond to collisions occurring in a strength or gravity dominated regime. The transition between the two regimes occurs in the range $100 \mathrm{~m} \leq R \leq 1 \mathrm{~km}$ for both ice and basalt. We note that for a given target size, $Q_{D}^{*}$ is a strong function of the projectile's angle of incidence. Differences in $Q_{D}^{*}$ between a head-on and a 75 degree impact reach about a factor 10 .

The increase of $Q_{D}^{*}$ in the gravitational regime is due to two factors. The most important is the fact that even though bodies exceeding $1 \mathrm{~km}$ in radius are almost entirely shattered (see section 4.3) in a $Q_{D}^{*}$ collision, the pieces do not all disperse because their relative velocities are smaller than the escape velocity of the aggregate. Hence, for catastrophically disrupted and dispersed bodies larger than $\approx 1 \mathrm{~km}$, all the largest remnants are found to be gravitationally bound aggregates of smaller fragments. The second effect is due

to gravitational shielding of the central region of the target (see section 3.2) but as we shall see in section 4.3 this affects only the largest targets in our 
mass range.

In many studies, one is not interested in the outcome of a particular collision but rather in the collisional evolution of an entire population. For these statistical studies, we can compute an effective threshold which is independent of angle of incidence, namely $Q_{D}^{*}(\alpha)$ averaged over all possible impact geometries but at fixed relative velocity. For an isotropic distribution of incoming projectiles (at infinity), Shoemaker (1962) showed that the probability distribution for impacting with an angle between $\alpha$ and $\alpha+d \alpha$ is given by

$$
P(\alpha) d \alpha=2 \sin (\alpha) \cos (\alpha) d \alpha \quad 0<\alpha<\pi / 2
$$

regardless of a planet's mass. Using this probability distribution, we define a mean catastrophic disruption threshold $\bar{Q}_{D}^{*}$ by

$$
\bar{Q}_{D}^{*}=\int_{0}^{\pi / 2} 2 Q_{D}^{*}(\alpha) \sin (\alpha) \cos (\alpha) d \alpha
$$

This integration is carried out numerically with a simple trapezoidal method and using the $Q_{D}^{*}(\alpha)$ determined from the simulations. The results are displayed in Fig. 6 for basalt targets and in Fig. 7 for icy targets.

In order to compare with disruption thresholds published in the literature and to allow these results to be used by others, we fitted (by eye) an analytical curve to $\bar{Q}_{D}^{*}$ of the following functional form

$$
\bar{Q}_{D}^{*}=Q_{0}\left(\frac{R_{p b}}{1 \mathrm{~cm}}\right)^{a}+B \rho\left(\frac{R_{p b}}{1 \mathrm{~cm}}\right)^{b}
$$

where $R_{p b}$ is the radius of the parent body (or target), $\rho$ the density of the parent body (in $\mathrm{g} / \mathrm{cm}^{3}$ ) and $Q_{0}, B, a, b$ constants to be determined. This functional form is often encountered in scaling law approaches with the two terms representing the two distinct physical regimes dominating the dynamics: 1) material strength (first term on the right, with $a<0$ ) and 2) self-gravity (second term on the right, with $b>0$ ). The values obtained for the coefficients are listed in Table 3 and the fits are represented as lines on Figs. 6 and 7 .

Note that the slopes in the gravity regime $(b)$ are somewhat different between basalt and ice, and even for ice the two slopes corresponding to the two velocities differ slightly. This could be related to the fact that for equal mass targets, ice material is lifted from an initially higher potential 
(less negative potential energy) corresponding to the larger equal-mass target diameter. Alternatively, because the shock imparts different velocities to fragments according to different material characteristics (the velocity field determines the subsequent gravitational reaccumulation), equation of state distinctions sensitive to both material type and impact speed may be the culprit.

Also note that in the gravity regime, faster impacts are less disruptive than slower impacts of the same energy, for both rock and ice. This is due to the greater efficiency of momentum coupling for slower impacts. The governing factor for gravity regime disruption is not shattering, but motion towards gravitational separation. For basalt, this trend continues into the strength regime, although that is coincidental, since the strength regime depends on entirely different aspects of collisional physics (flaw activation). For ice, the opposite is true: slower impacts (of the same energy) result in less disruption. Evidently ice is easier to fracture at high strain rates than at low strain rates, relative to basalt. This is either because it has more flaws available at low activation energies (consistent with its Weibull distribution), or because vaporization at high impact speed may contribute to fragmentation, which is not the case for the subsonic $(500 \mathrm{~m} / \mathrm{s})$ collisions.

Now we compare our averaged dispersion threshold with other published values in Fig. 8. In this figure, we reproduced the disruption thresholds obtained either from scaling laws or numerical simulations in the strength or gravitational dominated regime. For small targets, our estimate of the threshold agrees well with the determination of Holsapple (1994). The largest differences occur for large targets for which we predict that they are significantly stronger (more difficult to disrupt and disperse) than previously estimated. This does not arise due to a significant change of slope (notice our slope is close to the one predicted by Holsapple 1994 or Melosh \& Ryan 1997) but because the turn over from strength to gravity dominated targets occurs at smaller sizes.

We also display in Fig. 8 the recent determination of $Q_{D}^{*}$ by Durda et al. (1998). Their curve is determined by requiring that numerical models of the collisional evolution of the main belt asteroids fit the observed size distribution of these objects. Interestingly, they obtain that objects of order $100-200 \mathrm{~m}$ in diameter are the weakest objects, a conclusion confirmed by our simulations. However, besides the agreement on the size of the weakest objects, our results (as well as all other determinations of $Q_{D}^{*}$ ), differ from the results of Durda et al. by an order of magnitude or more. The origin 
of this discrepancy is not clear. On one hand, we note that the values of $Q_{D}^{*}$ determined by Durda et al. are not obtained from a simple fit to observed sizes but assume an underlying collisional model which might not be predicting accurately collisional outcomes. On the other hand, it may also be possible that asteroidal material has very different mechanical properties than the material tested in the laboratory.

As already noticed by many (most recently by Ryan and Melosh 1998), the efficiency at which the kinetic energy is transmitted from the impactor to individual fragments is extremely low. Since the largest remnants in collisions involving targets larger than $1 \mathrm{~km}$ are gravitationally bound rubble piles (see section 4.3) their mass is determined ultimately by the velocity distribution imparted to the fragments during the impact. A fragment will remain bound if its velocity remains below the escape velocity of the aggregate of all slow moving fragments. This explains why targets as small as $1 \mathrm{~km}$ radius are already significantly strengthened by gravity against dispersal.

For a given material type, the radius of the weakest object $R_{\text {weak }}$, is obtained by finding the radius for which $d \bar{Q}_{D}^{*} / d R=0$. From equation 6 the value of the radius of the weakest object is given by

$$
R_{\text {weak }}=\left(\frac{-B \rho b}{a Q_{0}}\right)^{\frac{1}{a-b}}
$$

Table 4 gives the values derived for $R_{\text {weak }}$ using the values of the parameters listed in Table 3. For both materials and impact speeds, $R_{\text {weak }} \simeq 100-200 \mathrm{~m}$.

These values are smaller than those derived in other studies. For example, Holsapple (1994) based on scaling laws gives $3 \mathrm{~km}$ as the transition point between the two regimes. Melosh \& Ryan (1997) as well as Love \& Ahrens (1996) from numerical simulations give numbers in the range $200-400 \mathrm{~m}$.

\subsection{Largest remnants}

While a collision occurring at $Q_{D}^{*}$ leaves by definition a largest remnant with mass equal to half the target mass, collisions occurring with different $Q$ leave remnants of different masses. We can therefore use all our simulations (whose intent was to bracket $Q_{D}^{*}$ ) to investigate how the mass of the largest remnant depends upon the incident kinetic energy per gram of target material $(Q)$.

Figs. 9 and 10 show the dependency of the mass of the largest fragment on the impact energy obtained in our simulations. To facilitate the 
interpretation of these results, the impact kinetic energy per gram of target material has been normalized to $Q_{D}^{*}$ for each target size and projectile angle of incidence. All simulations (120) involving a given material type and impact velocity have been plotted on the same plot. The different symbols correspond to targets of different sizes.

These figures clearly show that when normalized to $Q_{D}^{*}$ the relative mass of the largest remnant is a well-defined, simple function of $Q$ and is independent of target size and/or angle of incidence. The dependence upon these parameters enters only in $Q_{D}^{*}$ ! The increased scatter in the points for small mass fragments is probably due to the inherent numerical difficulties in resolving these smaller objects (at fixed resolution).

This relationship is remarkable since the mass of the largest remnant is not determined by a single process, but either by material strength or gravity depending on target size! In this regard it is interesting to note that in the case of low velocity collisions on icy targets the correlation is significantly worse, especially for large targets $(R \geq 3 \mathrm{~km})$. It is unclear why this is the case; however, in these cases it is worth pointing out that, due to the low velocity, the projectile is sometimes as big as the target and that the relative velocity is significantly smaller than sound speed, indicating that there might be a different disruption regime at low velocity.

Except for the case discussed above, we find that the relative mass of the largest remnant can be well represented by the following expression and that this single expression holds for targets ranging from $3 \mathrm{~cm}$ to $100 \mathrm{~km}$ and angles of incidence between 0 and 75 degrees.

$$
\frac{M_{\mathrm{lr}}}{M_{\mathrm{pb}}}=-s\left(\frac{Q}{Q^{*}}-1\right)+0.5
$$

These lines are drawn on the various figures and their slopes are remarkably similar: for basalt $s=0.35$ for $v=5 \mathrm{~km} / \mathrm{s}, s=0.5$ for $v=3 \mathrm{~km} / \mathrm{s}$ and for ice $s=0.6$ for $v=3 \mathrm{~km} / \mathrm{s}$. The case for ice at $v=0.5 \mathrm{~km} / \mathrm{s}$ for some still unknown reason does not yield such a tight relation. We have not attempted a real fit to the data and only plot the line derived for the $v=3 \mathrm{~km} / \mathrm{s}$.

\subsection{Largest intact fragments}

We now determine the largest intact (unshattered) fragment, $M_{\mathrm{lif}}^{*}$, in collisions occurring at $Q_{D}^{*}$. Here by undamaged we mean a fragment for which material strength still plays an important role in the cohesive properties of 
the object even though the fragment may no longer have its original strength. We are looking for the largest boulder in the final rubble-pile.

For each target radius and projectile angle of incidence, we obtain from the three simulations performed a parabolic relation $M_{\text {lif }}=f(Q)$. We determine $M_{\text {lif }}^{*}$ by setting $M_{\text {lif }}^{*}=f\left(Q_{D}^{*}\right)$ where $Q_{D}^{*}$ has been determined using the parabolic fit described in section 4.1. The values obtained for the mass of these largest intact fragments are displayed in Figs. 12 and 11 for both material types and impact velocities.

Not surprisingly, for small targets $(R \leq 100 \mathrm{~m})$ the largest undamaged fragment is equal to half the original target mass regardless of impact parameter. This is simply because in the strength dominated regime the mass of the largest intact fragment is equal to half the target mass by definition of $Q_{D}^{*}$. However, for targets larger than $300 \mathrm{~m}$ and regardless of material type and/or impact velocity, the mass of the largest intact fragment drops rapidly even though the collisions occurred at $Q_{D}^{*}$. This reflects the fact that collisions involving parent bodies this size and larger take place in the gravity dominated regime. This regime is therefore characterized by the fact that the largest remnant is not an intact fragment but a gravitationally bound aggregate of fragments. Due to the precipitous nature of these curves for target radii larger than a few $100 \mathrm{~m}$, we dont expect any monolithic object of this size. On the other hand, we expect a wide variety of internal structures for objects in the $30 \mathrm{~m}-300 \mathrm{~m}$ range.

For the largest targets considered in this study we notice a marginal trend for $M_{\text {lif }}^{*}$ to rise again. This effect is due to gravitational strengthening of the target discussed in section 3.2. However, we stress that this effect is only of moderate importance: the main role of gravity in this size range is to allow for the formation of gravitationally bound rubble piles. Gravitational strengthening appears also to be strongly dependent on initial impact parameter. For example, in the case of an icy target of $100 \mathrm{~km}$ radius a grazing impact occurring at $Q_{D}^{*}$ and with an incidence angle of $\alpha=75^{\circ}$ leaves behind a largest undamaged fragment of $\approx 0.015 M_{p b}$ while at $\alpha=0^{\circ}$ the same fragment is smaller than $0.003 M_{p b}$.

Final objects in the range $1 \mathrm{~km} \leq R_{p b} \leq 100 \mathrm{~km}$ are found to be essentially gravitationally bound aggregates of smaller fragments. Whether or not observed asteroids and comets in this size range are indeed rubble piles depends upon whether they have suffered a $Q_{D}^{*}$ collision during their history. Further complications exist; for example, Asphaug et al. (1998) have shown that target geometry (shape) and internal structure (pre-fracture) can sig- 
nificantly influence collisional outcome. The spherical homogeneous intact solids considered here may be idealized. A "contact binary" asteroid may for instance suffer catastrophic disruption of its impacted lobe, with little or no disruption occurring on its unimpacted lobe. A target which is already a rubble-pile may similarly be more difficult to disperse by impact, due to the inefficient coupling of impact energy.

\subsection{Ejection velocity of largest remnants}

For each target size, material type and impact parameter we determined the ejection velocity of the largest mass remnant in each of the three simulations bracketing $Q_{D}^{*}$. We note that this velocity is not related in a straightforward manner to the usual energy partitioning coefficient, $f_{\mathrm{KE}}$, namely the fraction of kinetic energy going into fragment kinetic energy. Given that the kinetic energy is not distributed uniformly over all fragments, but rather carried away by a small amount of mass moving very fast, we believe the coefficient $f_{\mathrm{KE}}$ to be of little use to address the dynamics of the largest fragments. We therefore focus our attention on determining the actual velocity of the largest fragment or aggregate in each simulated collision, numerical resolution preventing us from studying the smaller ones.

These ejection velocities as a function of normalized fragment mass are displayed in Figs.(13, 14, 15, 16) for both material types and collision velocities. Velocity is measured relative to the center of mass of the original target.

In each figure, the upper panel shows the actual ejection velocity. Each different symbol corresponds to a different initial target size regardless of angle of incidence. The lower panel shows the ejection velocity normalized to the target's escape velocity for initial targets greater that $1 \mathrm{~km}$.

We note that for a given parent body size, larger remnants have lower ejection velocities regardless of angle of incidence. In fact, it is remarkable how little influence the impact parameter seems to have on the ejection velocity of the largest remnant. For each target size, the ejection velocity of the largest remnant is to a good approximation a simple decreasing linear function of its fractional mass (in the domain $0.15 \leq M_{l r} / M_{p b} \leq 0.8$ ). In addition, we note that for the largest fragments, i.e. the one for which gravity is the dominant cohesion force, we can almost remove the target size dependence by normalizing the ejection velocity by the parent body's escape velocity. In other words, the outcome velocity of the largest fragment nor- 
malized to initial target escape velocity is (within some considerable scatter) independent of target size and impact parameter. The fact that the velocity of the largest remnant is a relatively constant fraction of the target's escape velocity is probably due to the a priori requirement that in the gravitational regime about half of the initial mass must escape.

In order to gain insight regarding which collisions lead to the fastest moving largest remnants, we have analyzed how the ejection velocities depend upon the ratio of impactor to target size, $R_{i} / R_{p b}$. We stress again that because of numerical resolution, we are able to analyze only the largest remnants and not the entire ejecta distribution. Thus, it is unrealistic from our data to determine the actual kinetic energy transfer efficiency $f_{\mathrm{KE}}$.

Figs. 17 and 18 display the ejection velocity of the largest remnants as a function of $R_{i} / R_{p b}$ regardless of angle of incidence. The two different symbols correspond in each cases to the two different impact velocities. Apparent from these figures is the fact that the ejection velocity rises with $R_{i} / R_{p b}$ in a monotonic fashion. The "width" of the curve is mainly determined not by scatter but by the relation between ejection velocity and remnant mass (see section(4.4). Thus, regardless of angle of incidence, collisions will give rise to fast moving remnants if the size of the impactor becomes comparable to the size of the parent body.

In regard to the collisional origin of asteroid families, we note that velocities of order $100 \mathrm{~m} / \mathrm{s}$ are easily obtained for basaltic targets provided the impactor size is at least about half the parent body size.

\section{Conclusions}

We have presented a self-consistent three-dimensional treatment of impact disruption leading from the laboratory and the strength regime, where our SPH code has been exhaustively calibrated and tested, all the way out to the gravity regime collisions responsible for the formation of asteroid families and planetary accumulation. While some parameters (such as shape and pre-fragmentation and rotation) have yet to be fully explored (see for instance Asphaug et al. 1998) the 480 runs summarized here provide a robust constraint on the outcome of catastrophic collisions.

In particular, we have demonstrated that bodies 100 - $200 \mathrm{~m}$ radius (depending on impact speed and composition; see Table 4) are the weakest objects in the solar system, with all bodies this size and larger being domi- 
nated by self-gravitational forces, rather than material strength, with regard to impact disruption. This enhanced role of gravity is not due as usually assumed to fracture prevention by gravitational compression. It is due to the difficulty of the fragments to escape their mutual gravitational attraction. Owing to the generally low efficiency of momentum transfer in collisions, the velocity of larger fragments tends to be small, and more energetic collisions are needed to disperse them. Remarkably, the efficiency of momentum transfer (while still small) is found to be larger for larger projectiles. Thus, at a fixed collisional energy, a low velocity high mass projectile will lead to a higher fragment velocity that a small mass high speed projectile.

This increased role of gravity implies that the threshold for disruption is actually significantly larger than previously assumed. The upshot of this is that any catastrophic collisions leading to disruption must occur at an energy far exceeding the threshold for shattering the parent body. These necessarily high strain rate collisions imply by the nature of the fracturing process that many cracks must grow to release the stresses, preventing any sizeable fragment from surviving. Thus, catastrophic collisions of this nature can only result in the formation of gravitationally bound aggregates of smaller fragments.

We shall continue to examine the outcome of these simulations for information regarding angular momentum transfer during impact, and for anticipated cumulate structures for large fragments from large parent bodies, motivated by the possibility of re-creating the events which led to the formation of known asteroid families (Benz and Michel, in preparation). As available computing power permits, a more detailed parameteric exploration (varying Weibull coefficients, shape and internal structure) is someday hoped for. As it stands, our chosen materials (basalt and ice) represent broad-based choices, and the fact that both give similar results implies that catastrophic disruption is perhaps not very material dependent. If that is the case, then the simple and robust relations presented here, for the mass of the largest remnant in an impact event, and for the ejection velocity of the largest remnant, are appropriate for a new generation of calculations modeling the accretion of planets in ours and other solar systems.

This work was supported in part by the Swiss National Science Foundation and by NASA grant NAG5-7245 from the Planetary Geology and Geophysics Program. 


\section{References}

Ahrens, T.J., and Rubin, A.M., 1993, Impact-induced tensional failure in rock, J. Geophys. Res., 98, 1185-1203.

Asphaug, E. and H. J. Melosh (1993). The Stickney impact of Phobos: a dynamical model. Icarus 101, 144-164

Asphaug, E., 1997, Impact origin of the Vesta family, Meteor. and Plan. Sci., 32, 965-980.

Asphaug, E., S.J. Ostro, R.S. Hudson, D.J. Scheeres and W. Benz, 1998, Disruption of kilometre-sized asteroids by energetic collisions, Nature, 393, 437-440.

Benz, W., 1989, Smooth Particle Hydrodynamics: a Review, in Numerical Modeling of Nonlinear Stellar Pulsations. Problems and Prospects, ed. J.R. Buchler, Dordrecht: Kluwer Academic Press, p. 269-288.

Benz, W., and E. Asphaug, 1994, Impact simulations with fracture. I. Method and tests, Icarus, 107, 98-116.

Benz, W., and E. Asphaug, 1995, Simulations of brittle solids using smooth particle hydrodynamics Comput. Phys. Comm., 87, 253-265.

Davis, D.R., \& Ryan, E.V., 1990, On collisional disruption - Experimental results and scaling laws, Icarus 83, 156.

Davis, D.R., Ryan, E.V., and P. Farinella, 1997, On how to scale disruptive collisions, Lun. Plan. Science, XXVI, 319-320.

Durda, D.D., Greenberg, R., and R. Jedicke, 1998, Collisional models and scaling laws: A new interpretation of teh shape of the main-belt asteroid size distribution, Icarus, 135, 431-440.

Fujiwara, A., Cerroni, P, Davis, D.R., Ryan, E.V., DiMartino, M., Holsapple, K.A., and Housen, K.R., 1989, in Asteroids II, eds. R.P. Binzel, T. Gehrels, M.S. Matthews, University of Arizona Press, Tucson.

Holsapple, K.A., 1994, Catastrophic disruptions and cratering of solar system bodies: A review and new results, Plan. Spac. Science, 42, 10671078.

Holsapple, K. A., and K. R. Housen, 1986, Scaling laws for the catastrophic collisions of asteroids, Mem. S.A.It. 57, 65-85

Housen, K.R., and K.A. Holsapple, 1990, On the fragmentation of asteroids and planetary satellites, Icarus, 84, 226-253.

Housen, K.R., 1991, Laboratory simulations of large-scale fragmentation events, Icarus 94, 180-190. 
Housen, K.R. \& K.A. Holsapple, 1999, Scale effects in strength-dominated collisions of rocky asteroids, Icarus, this issue.

Jaeger, J.C., and N.G.W. Cook, 1969, Fundamentals of Rock Mechanics, (London:Chapman and Hall).

Lange, M.A. and T.J. Ahrens, 1983, The dynamic tensile strength of ice and ice-silicate mixtures, J. Geophys. Res 88, 1197-1208.

Love, S.G., and Ahrens, T.J., 1996, Catastrophic impacts on gravity dominated asteroids, Icarus, 124, 141-155.

Melosh, H.J., 1989, Impact Cratering: A Geologic Process, (New York: Oxford University Press).

Melosh, H.J., E. Ryan, and E. Asphaug (1992). Dynamic fragmentation in impacts. J. Geophys. Res. 97: 14,735-14,759.

Melosh, H.J., and Ryan, E.V., 1997, Asteroids: Shattered not dispersed, Icarus, 129, 562-564.

Monaghan, J.J., 1992, Smooth Particle Hydrodynamics. Ann. Rev. Astron. Astrophys., 30, 543-574.

Nakamura, A., and A. Fujiwara, 1991, Velocity distribution of fragments formed in a simulated collisional disruption, Icarus, 92, 132-146.

O'Keefe, J.D. and T.J. Ahrens, 1982a, The interaction of the Cretaceous/Tertiary extinction bolide with the atmosphere, ocean and solid earth. Geol. Soc. Amer. Special Papers 190, 103-120.

O'Keefe, J.D. and T.J. Ahrens, 1982b, Cometary and meteorite swarm impact on planetary surfaces, J. Geophys. Res. 87, 6668-6680.

Ryan, E.V., Hartmann, W.K., \& Davis, D.R., Impact experiments III Catastrophic fragmentation of aggregate targets and relation to asteroids, 1991, Icarus 94, 283-298

Ryan, E.V., and Melosh, H.J., 1998, Impact fragmentation: From the laboratory to asteroids, Icarus, 133, 1-24

Shoemaker, E. M., 1962, Interpenetration of lunar craters. In Z. Kopal (Ed.), Physics and Astronomy of the Moon, Academic Press, New York and London, pp. 283-359.

Tillotson, J. H., 1962, Metallic equations of state for hypervelocity impact, Rep. GA-3216, July 18, Gen. At., San Diego, California

Weibull, W. A., 1939, A statistical theory of the strength of materials (transl.), Ingvetensk. Akad. Handl., 151 (Stockholm), 5-45. 
Table 1: Material-dependent constants

\begin{tabular}{lllllll}
\hline \hline & $\mu$ & $\mathrm{Y}$ & $E_{\text {melt }}$ & $\mathrm{k}$ & $\mathrm{m}$ \\
& $\mathrm{erg} / \mathrm{cc}$ & $\mathrm{erg} / \mathrm{g}$ & $\mathrm{erg} / \mathrm{g}$ & $\mathrm{cm}^{-3}$ & & \\
\hline basalt & $2.2710^{11}$ & $3.510^{10}$ & $3.410^{10}$ & $4.010^{29}$ & $a$ & $9.0^{a}$ \\
ice & $2.8010^{10}$ & $1.010^{10}$ & $7.010^{9}$ & $1.410^{32}$ & ${ }^{b}$ & $9.6^{b}$ \\
\hline \hline
\end{tabular}

${ }^{a}$ From calibrating numerical simulations to laboratory experiments; Benz \& Asphaug (1995)

${ }^{b}$ Our fit to stress-strain rate data published in Lange \& Ahrens (1983) 
Table 2: Tillotson eos parameters

\begin{tabular}{lllllllllll}
\hline \hline & $\rho_{0}$ & $\mathrm{~A}$ & $\mathrm{~B}$ & $E_{0}$ & $\mathrm{E}_{\mathrm{iv}}$ & $\mathrm{E} \mathrm{E}_{\mathrm{cv}}$ & $\mathrm{a}$ & $\mathrm{b}$ & $\alpha$ & $\beta$ \\
& $\mathrm{g} / \mathrm{cc}$ & $\mathrm{erg} / \mathrm{cc}$ & $\mathrm{erg} / \mathrm{cc}$ & $\mathrm{erg} / \mathrm{g}$ & $\mathrm{erg} / \mathrm{g}$ & $\mathrm{erg} / \mathrm{g}$ & & & & \\
\hline basalt & 2.7 & $2.6710^{11}$ & $2.6710^{11}$ & $4.8710^{12}$ & $4.7210^{10}$ & $1.8210^{11}$ & 0.5 & 1.50 & 5.0 & 5.0 \\
ice & 0.917 & $9.4710^{10}$ & $9.4710^{10}$ & $1.0010^{11}$ & $7.7310^{9}$ & $3.0410^{10}$ & 0.3 & 0.1 & 10.0 & 5.0 \\
\hline \hline
\end{tabular}

${ }^{a}$ Tillotson parameters as published for lunar gabbroic anorthosite (O'Keefe \& Ahrens 1982a), substituting the basalt reference density and bulk modulus as reported by Nakamura \& Fujiwara (1991), from whose work our basalt fracture coefficients (Table 1) are derived.

${ }^{b}$ Tillotson parameters as expressed for water ice (O'Keefe \& Ahrens 1982b), again substituting the ice reference density and bulk modulus as reported by Lange \& Ahrens (1983), from whose work our ice fracture coefficients are derived. 
Table 3: Fit constants for $\bar{Q}_{D}^{*}$

\begin{tabular}{cccccc}
\hline \hline material & $\begin{array}{c}v_{\text {impact }} \\
\mathrm{km} / \mathrm{s}\end{array}$ & $\begin{array}{c}Q_{0} \\
\mathrm{erg} / \mathrm{g}\end{array}$ & $\begin{array}{c}\mathrm{B} \\
\mathrm{erg} \mathrm{cm} / \mathrm{g}^{2}\end{array}$ & $\mathrm{a}$ & $\mathrm{b}$ \\
\hline basalt & 5 & $9.010^{7}$ & 0.5 & -0.36 & 1.36 \\
basalt & 3 & $3.510^{7}$ & 0.3 & -0.38 & 1.36 \\
ice & 3 & $1.610^{7}$ & 1.2 & -0.39 & 1.26 \\
ice & 0.5 & $7.010^{7}$ & 2.1 & -0.45 & 1.19 \\
\hline \hline
\end{tabular}


Table 4: Radius of the weakest object

\begin{tabular}{ccc}
\hline \hline material & $\begin{array}{c}v_{\text {impact }} \\
\mathrm{km} / \mathrm{s}\end{array}$ & $\begin{array}{c}R_{\text {weak }} \\
\mathrm{m}\end{array}$ \\
\hline basalt & 5 & 163 \\
basalt & 3 & 117 \\
ice & 3 & 102 \\
ice & 0.5 & 213 \\
\hline \hline
\end{tabular}




\section{Figure Captions}

Figure 1: In the strength dominated regime, the largest remnant is a single intact fragment while in the gravity dominated regime the largest remnant is a gravitationally bound aggregate of fragments of various sizes.

Figure 2: Catastrophic disruption thresholds for a basalt target and $3 \mathrm{~km} / \mathrm{s}$ impact velocity. Each set of connected dots represent one projectile angle of incidence starting with 0 (bottom curve), 30, 45, 60, and 75 (top curve) degrees.

Figure 3: Catastrophic disruption thresholds for a basalt target and $5 \mathrm{~km} / \mathrm{s}$ impact velocity. Each set of connected dots represent one projectile angle of incidence starting with 0 (bottom curve), 30, 45, 60, and 75 (top curve) degrees.

Figure 4: Catastrophic disruption thresholds for an icy target and $3 \mathrm{~km} / \mathrm{s}$ impact velocity. See Fig(2) for caption

Figure 5: Catastrophic disruption thresholds for an icy target and $0.5 \mathrm{~km} / \mathrm{s}$ impact velocity. See Fig(2) for caption 
Figure 6: Mean catastrophic disruption threshold $\bar{Q}_{D}^{*}$ for a random distribution of impact parameters (see text for details) in the case of basalt targets

Figure 7: Mean catastrophic disruption threshold $\bar{Q}_{D}^{*}$ for a random distribution of impact parameters (see text for details) in the case of icy targets

Figure 8: Comparison between the mean catastrophic disruption threshold $\bar{Q}_{D}^{*}$ (basalt targets, $v=3 \mathrm{~km} / \mathrm{s}$ ) determined in this work (heavy line) and other determinations in the literature

Figure 9: Mass of largest remnant (in terms of original target mass) as a function of impact energy per gram of target material normalized to $Q_{D}^{*}$ for icy targets and collisions occurring at a) $0.5 \mathrm{~km} / \mathrm{s} \mathrm{b}$ ) $3 \mathrm{~km} / \mathrm{s}$. Different symbols correspond to different target sizes

Figure 10: Mass of largest remnant (in terms of original target mass) as a function of impact energy per gram of target material normalized to $Q_{D}^{*}$ for basaltic targets and collisions occurring at a) $3 \mathrm{~km} / \mathrm{s} \mathrm{b}) 5 \mathrm{~km} / \mathrm{s}$. Different symbols correspond to different target sizes

Figure 11: The mass of the largest intact fragment (normalized to mass of the parent body) when the threshold critical disruption occurs $\left(Q_{D}^{*}\right)$ for basalt targets. When gravity goes to zero, $M_{\text {lif }}$ goes to 0.5 by definition. The results for different angles of incidence are shown by using different symbols 
Figure 12: The mass of the largest intact fragment (normalized to mass of the parent body) when the threshold critical disruption occurs $\left(Q_{D}^{*}\right)$ for icy targets. When gravity goes to zero, $M_{l i f}$ goes to 0.5 by definition. The results for different angles of incidence are shown by using different symbols

Figure 13: Ejection velocities of largest remnant in $5 \mathrm{~km} / \mathrm{s}$ collisions involving basalt targets. Different symbols correspond to different parent body sizes. In the lower panel, the velocities have been normalized to the parent body's escape velocity and only the results for initial bodies with $R_{p b} \geq 1 \mathrm{~km}$ are shown

Figure 14: Ejection velocities of largest remnant in $3 \mathrm{~km} / \mathrm{s}$ collisions involving basalt targets. Different symbols correspond to different parent body sizes. In the lower panel, the velocities have been normalized to the parent body's escape velocity and only the results for initial bodies with $R_{p b} \geq 1 \mathrm{~km}$ are shown

Figure 15: Ejection velocities of largest remnant in $3 \mathrm{~km} / \mathrm{s}$ collisions involving icy targets. Different symbols correspond to different parent body sizes. In the lower panel, the velocities have been normalized to the parent body's escape velocity and only the results for initial bodies with $R_{p b} \geq 1 \mathrm{~km}$ are shown

Figure 16: Ejection velocities of largest remnant in $0.5 \mathrm{~km} / \mathrm{s}$ collisions involving icy targets. Different symbols correspond to different parent body sizes. In the lower panel, the velocities have been normalized to the parent body's escape velocity and only the results for initial bodies with $R_{p b} \geq 1 \mathrm{~km}$ are shown 
Figure 17: Ejection velocities of largest remnant as a function of impactor radius normalized to target radius in collisions involving basalt targets.

Figure 18: Ejection velocities of largest remnant as a function of impactor radius normalized to target radius in collisions involving icy targets. 
Strength regime:

Individual fragments

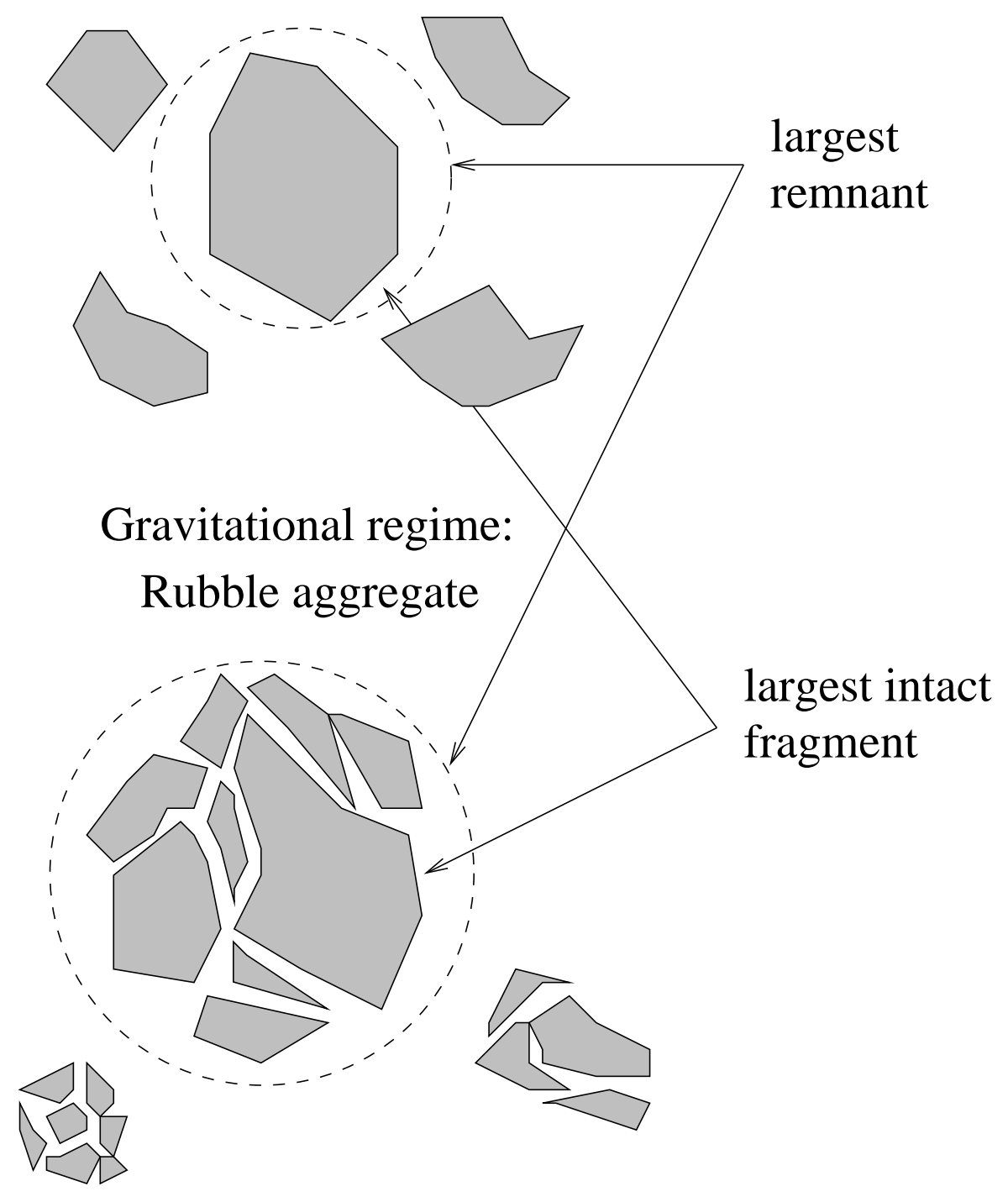

Figure 1: 


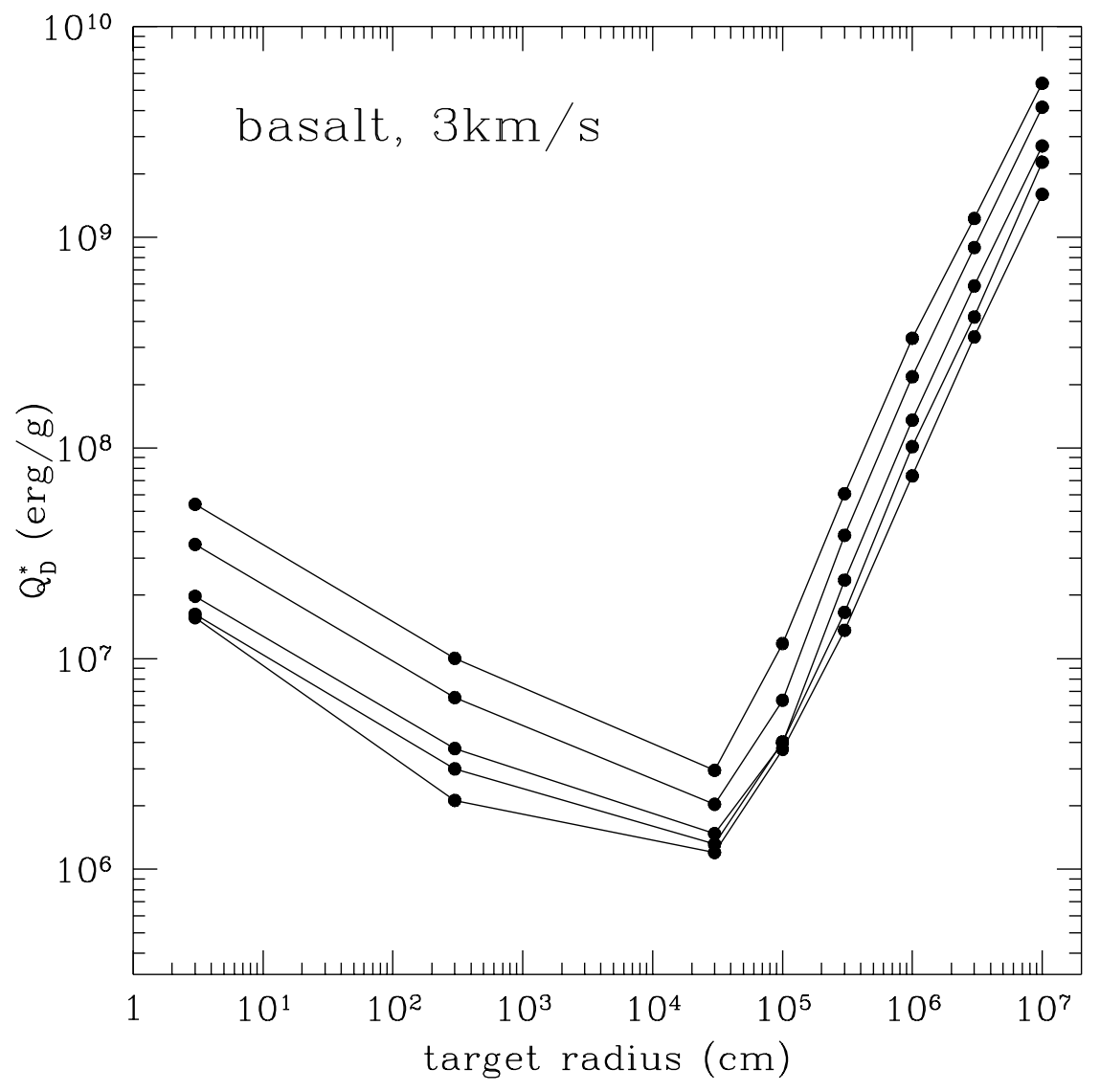

Figure 2: 


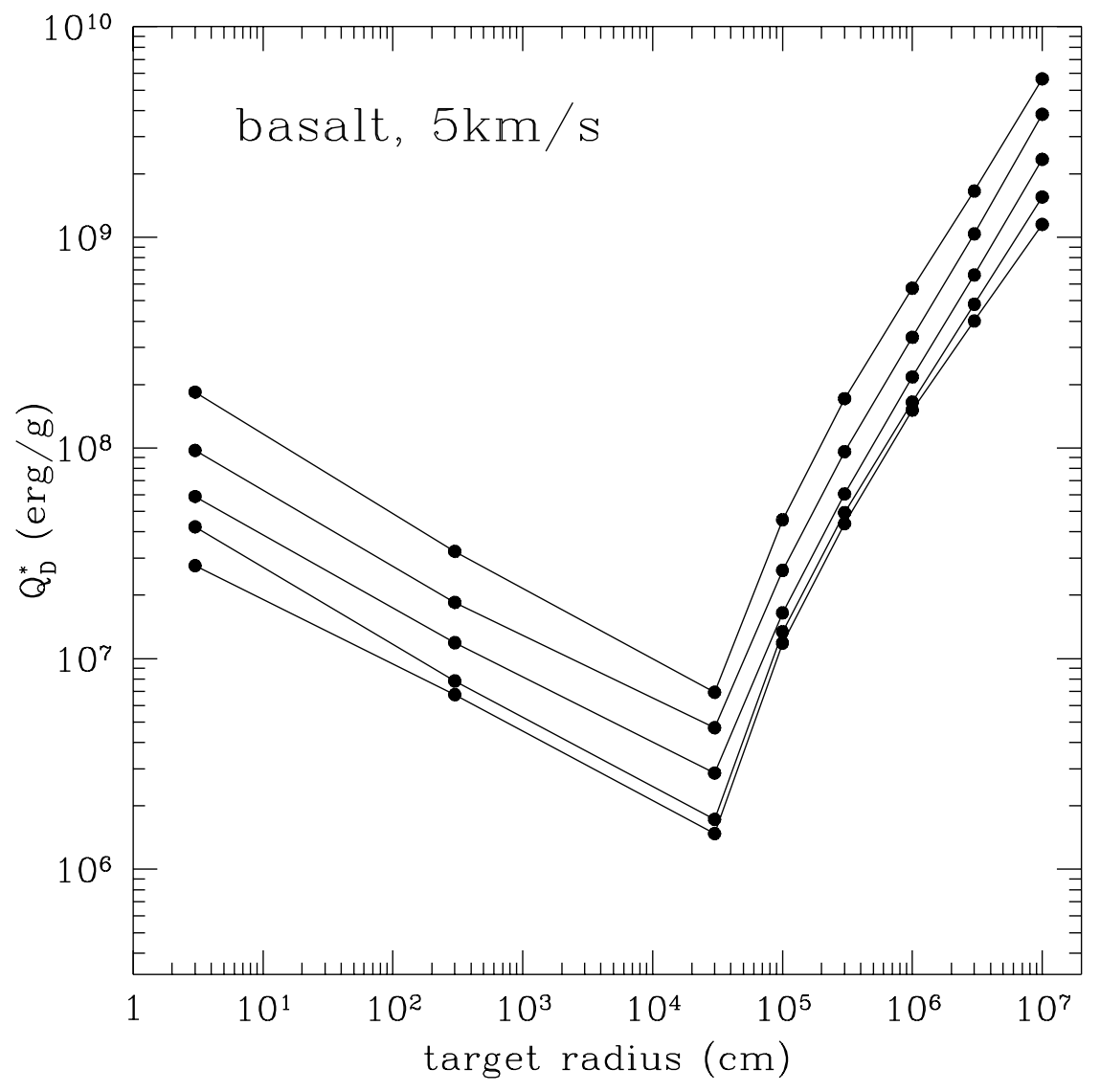

Figure 3: 


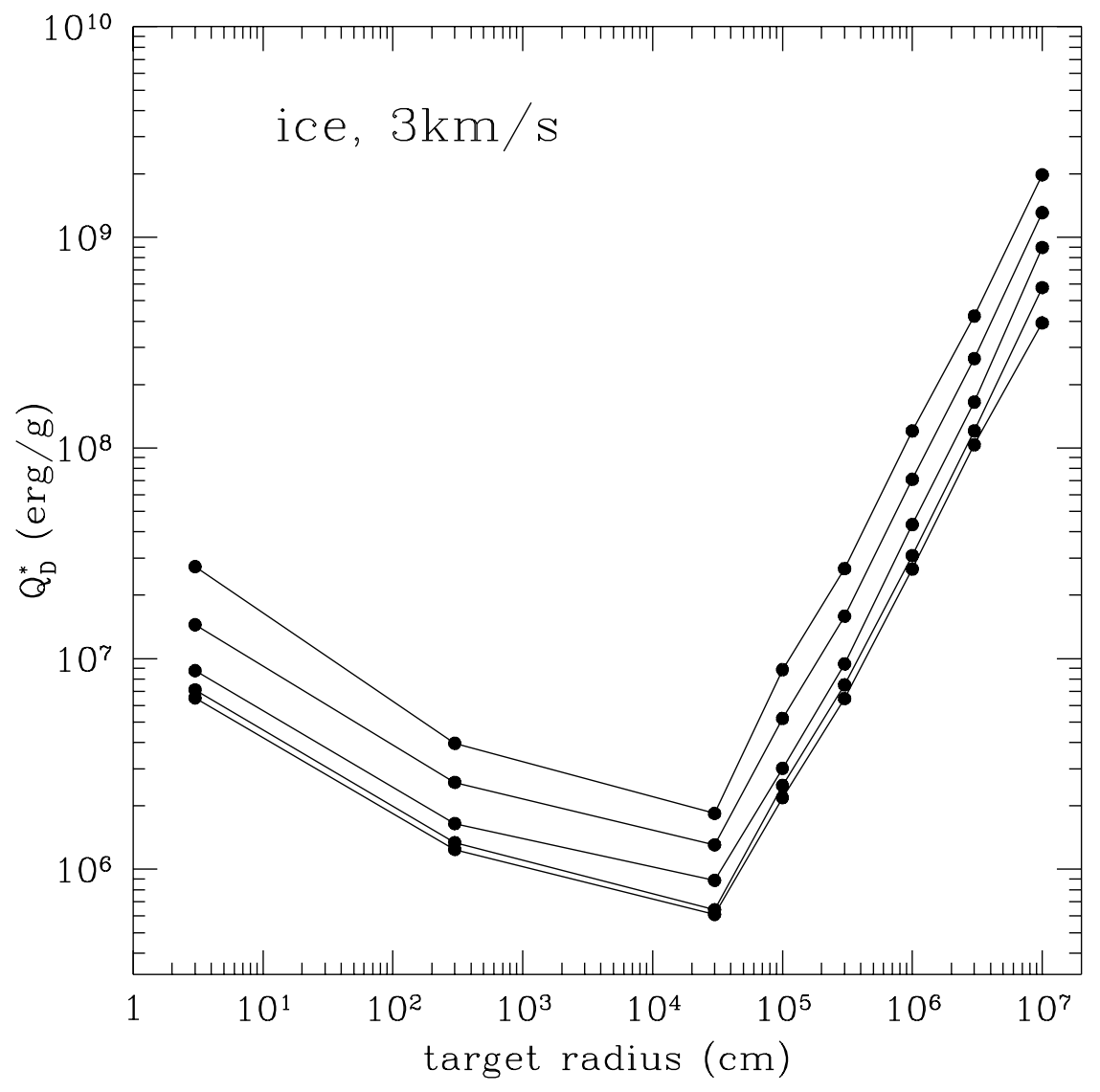

Figure 4: 


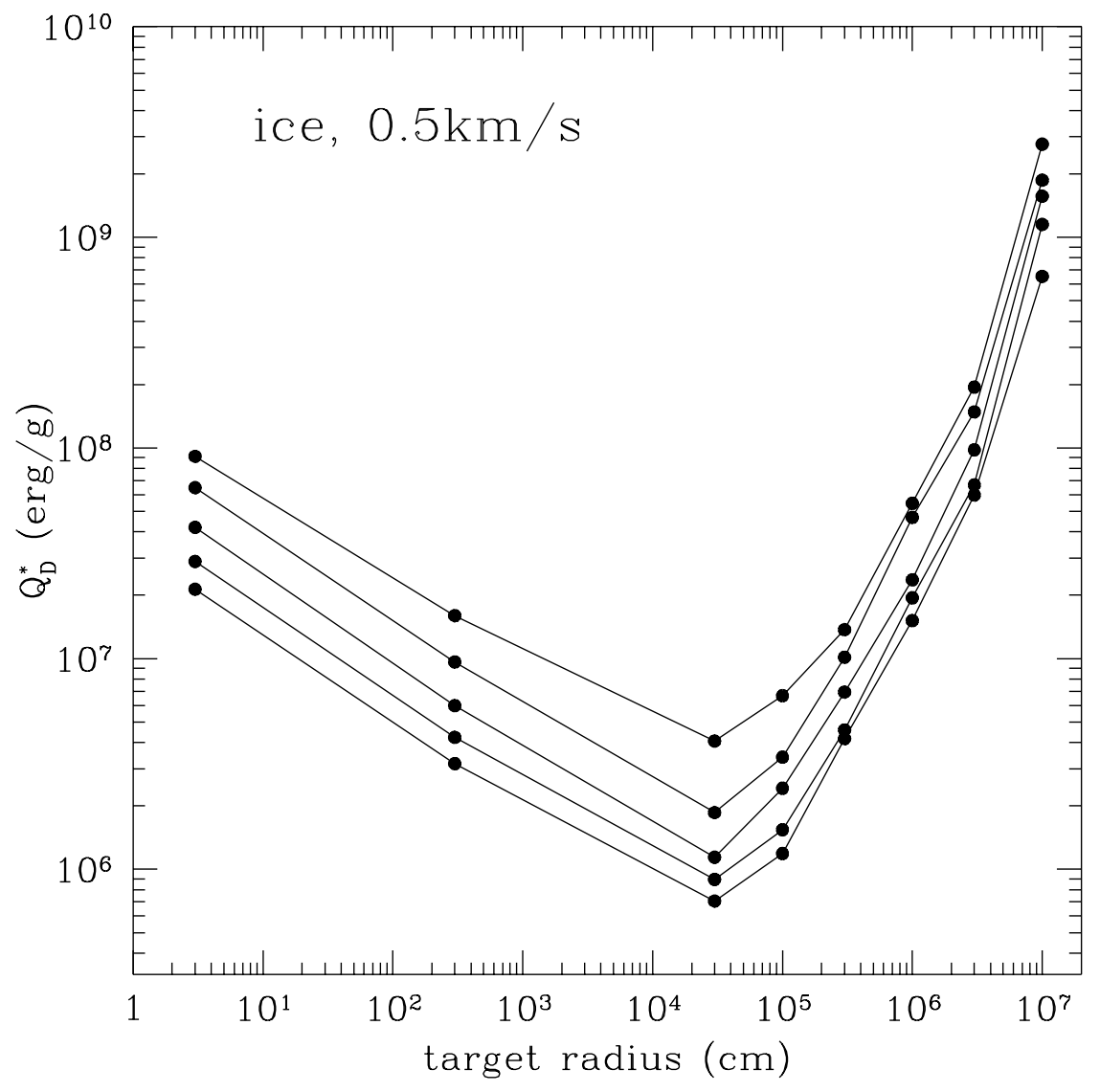

Figure 5: 


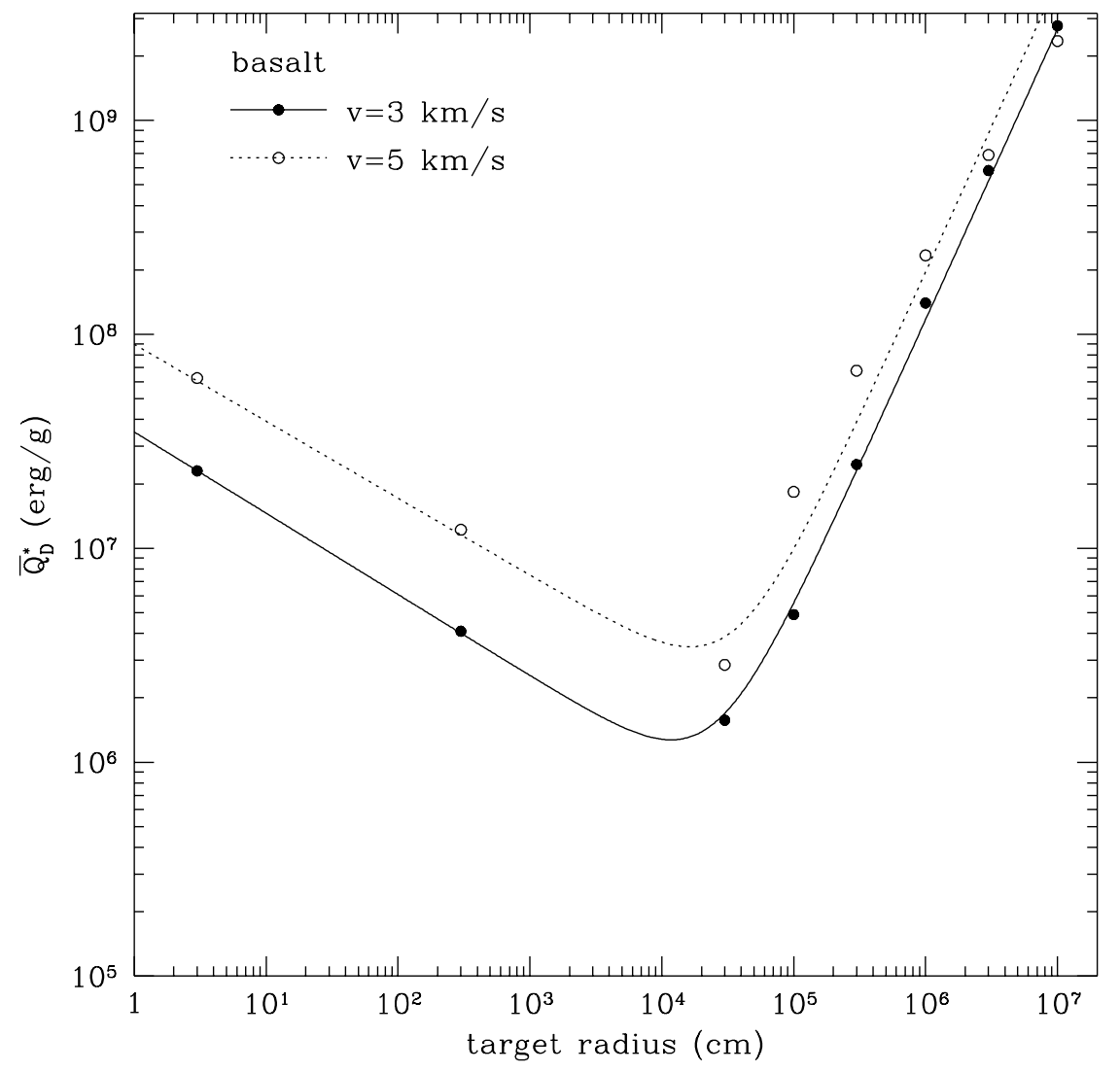

Figure 6: 


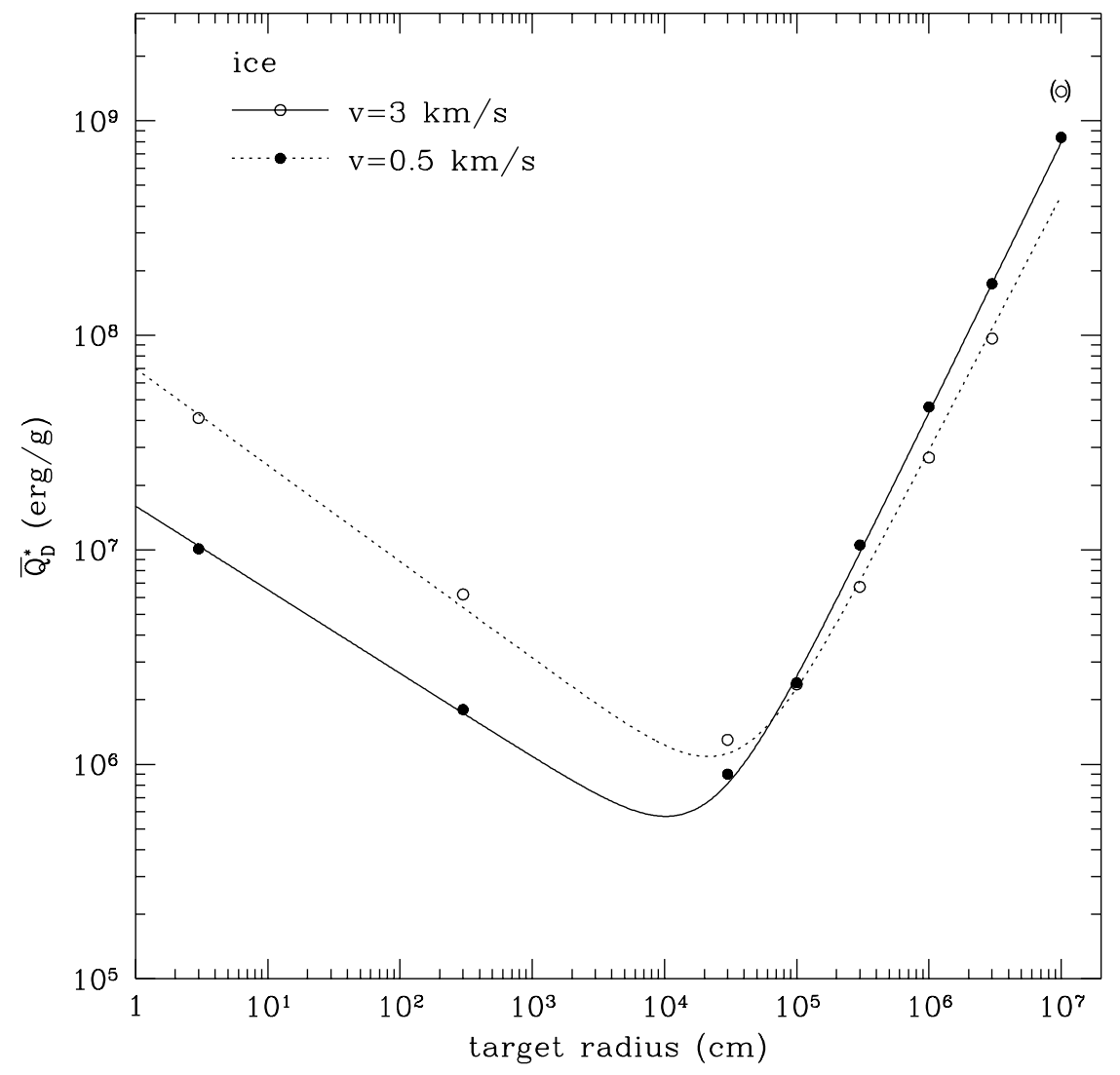

Figure 7: 


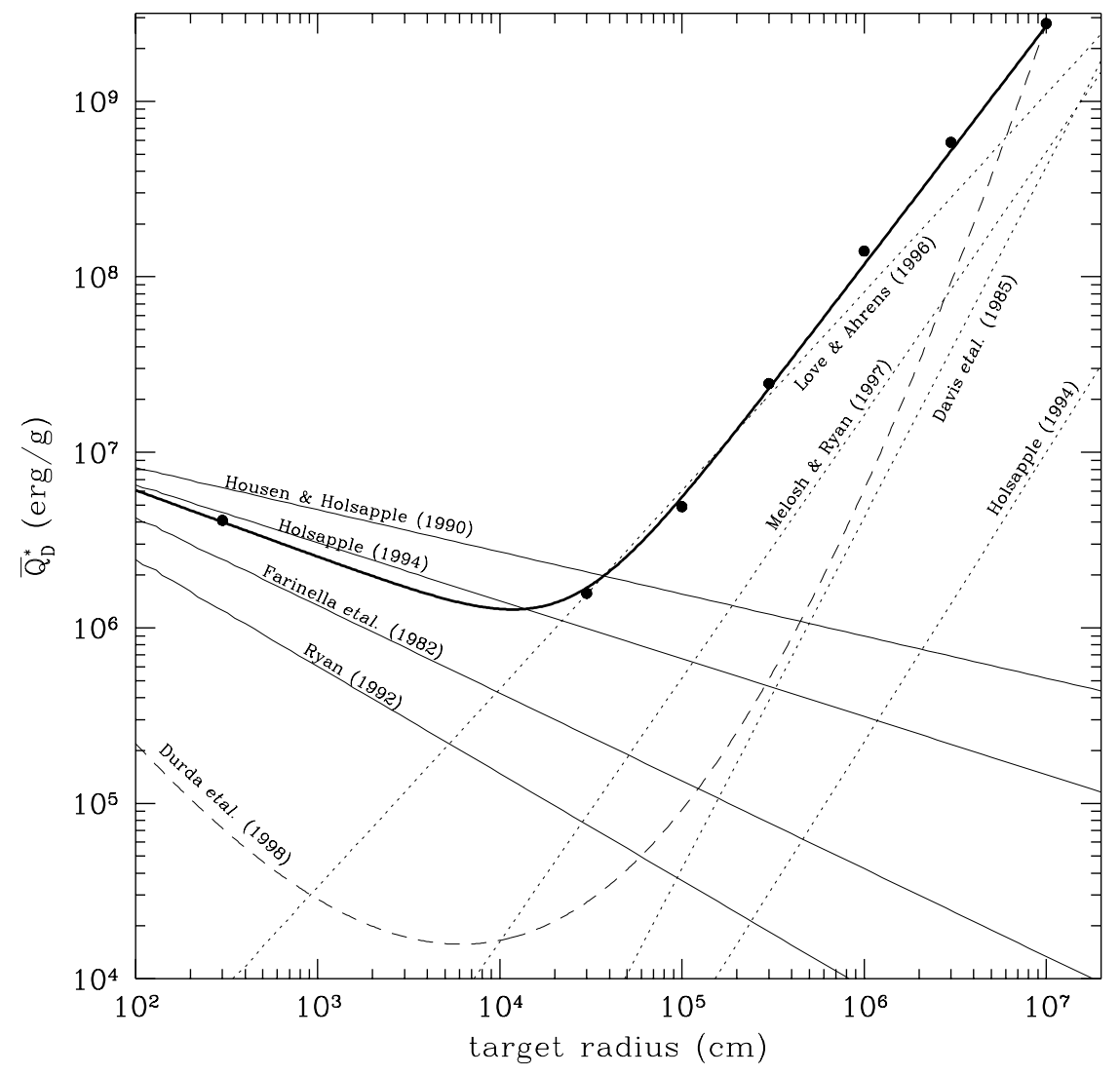

Figure 8: 

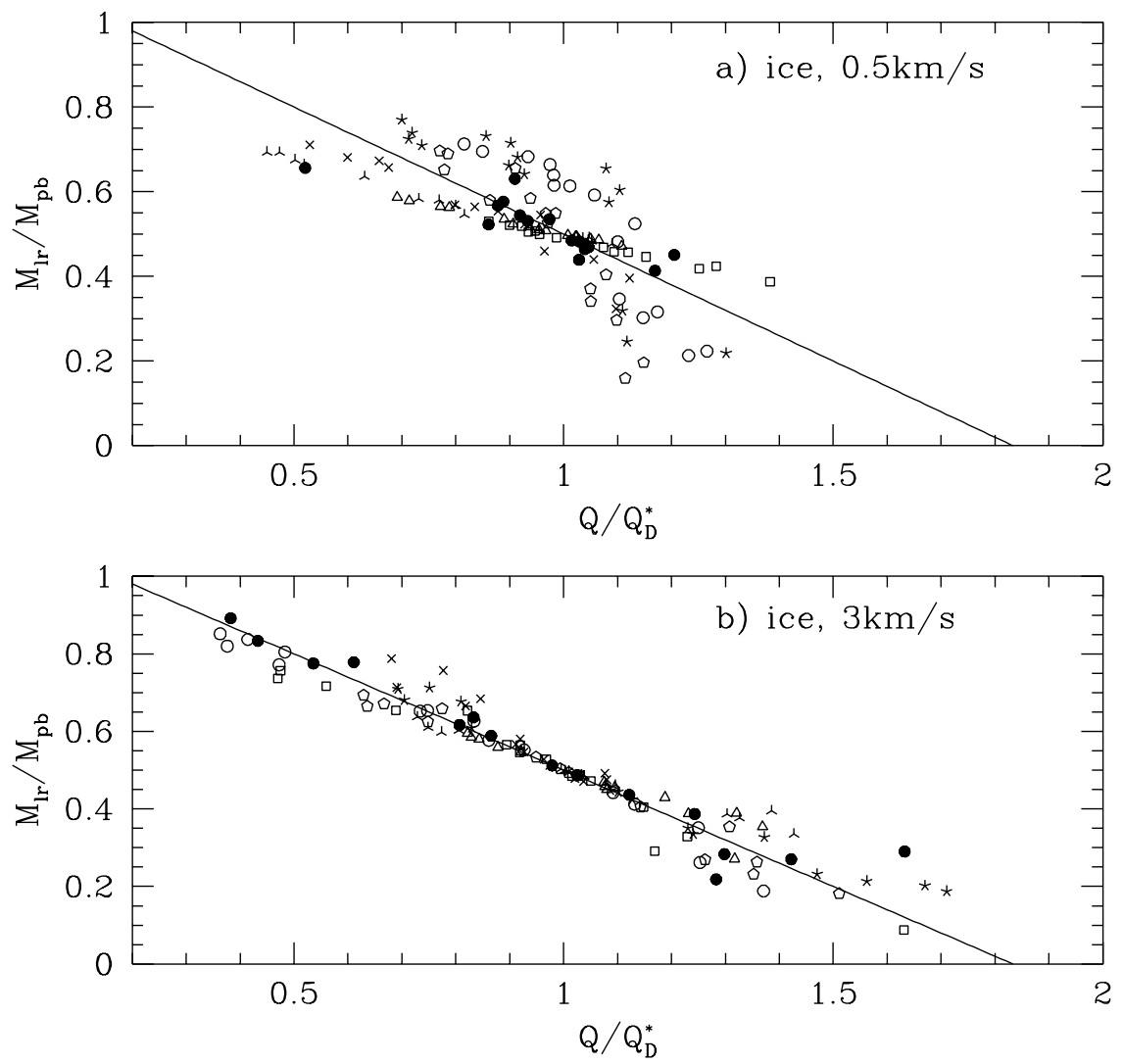

Figure 9: 

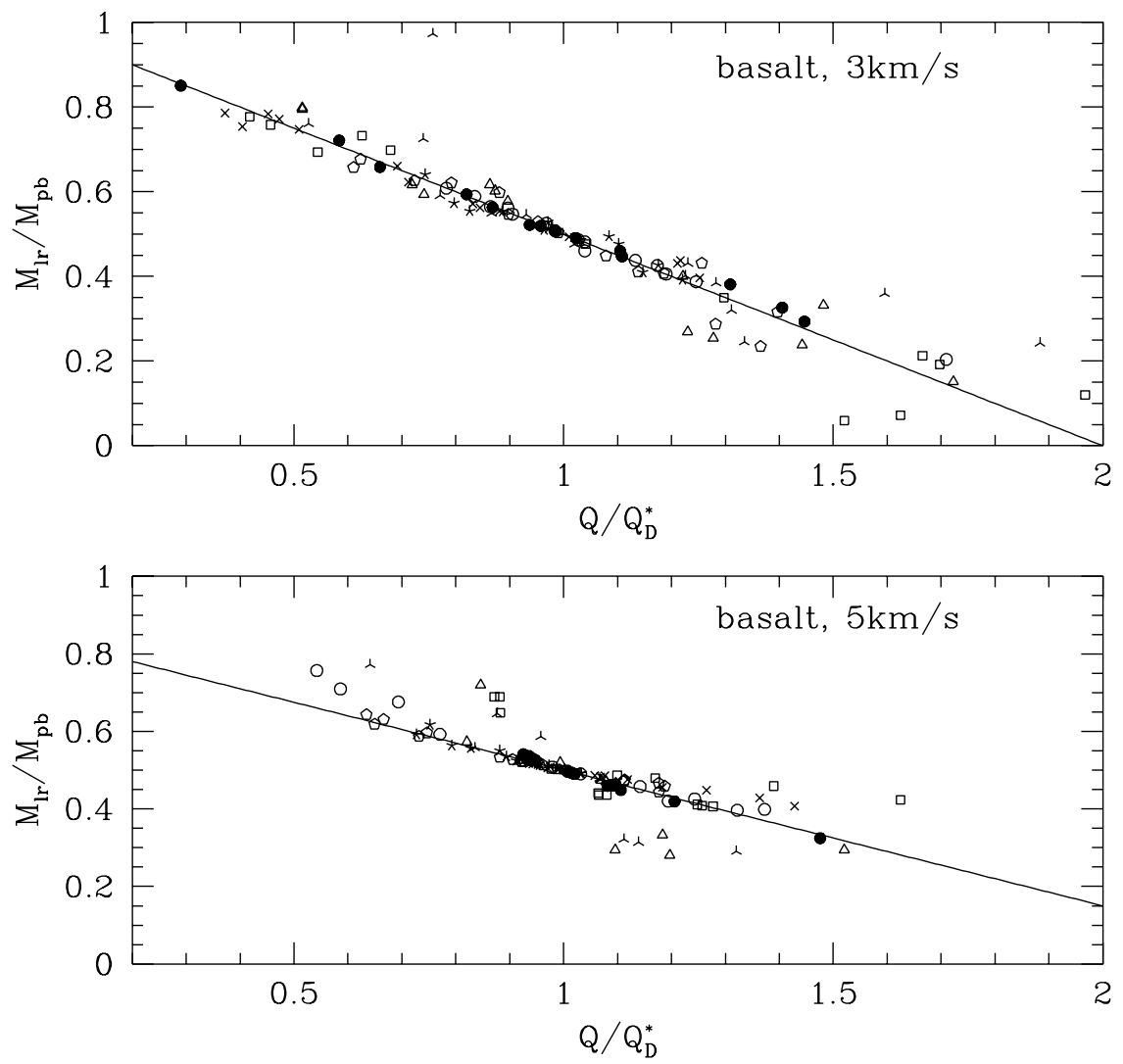

Figure 10: 

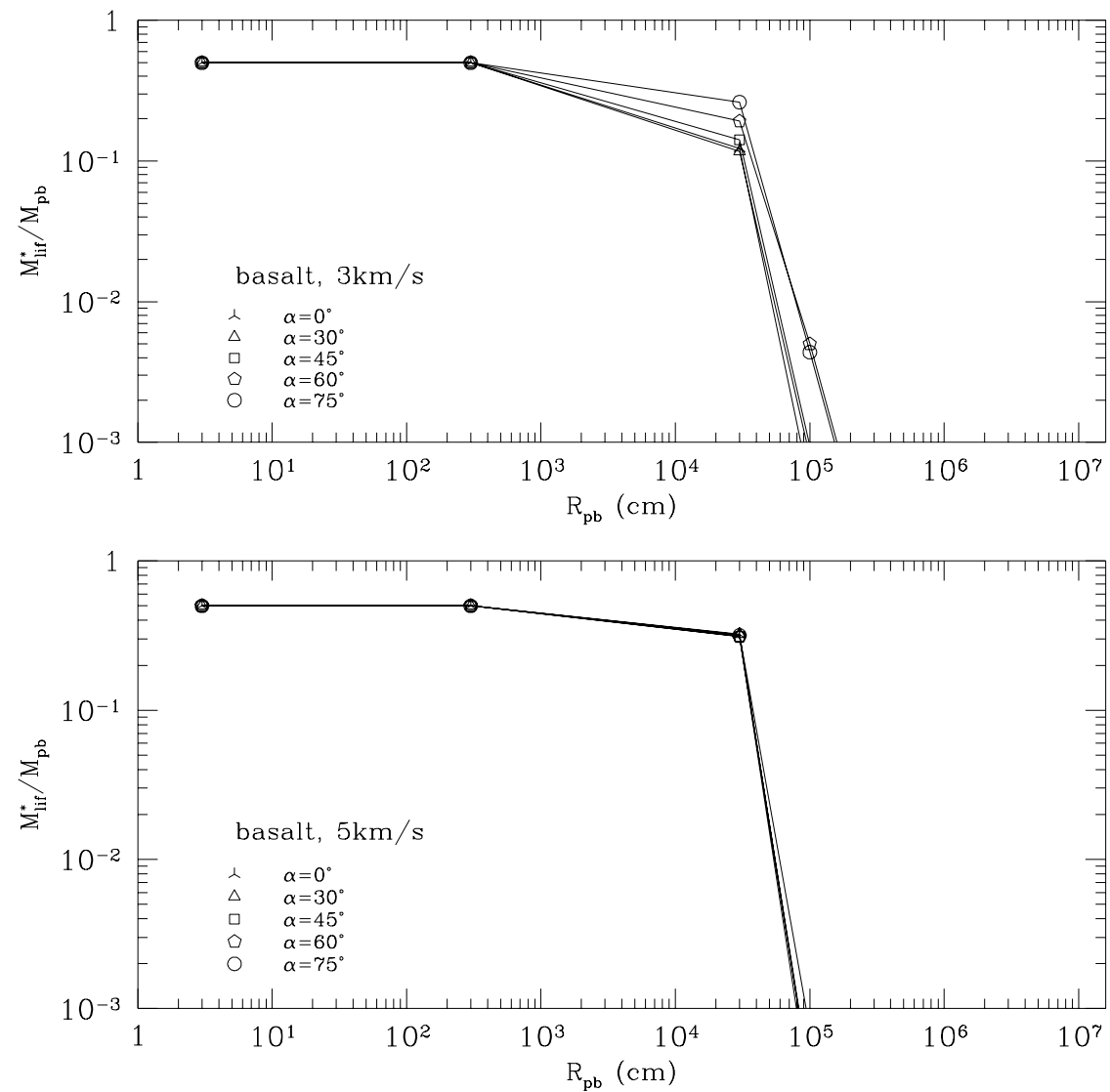

Figure 11: 

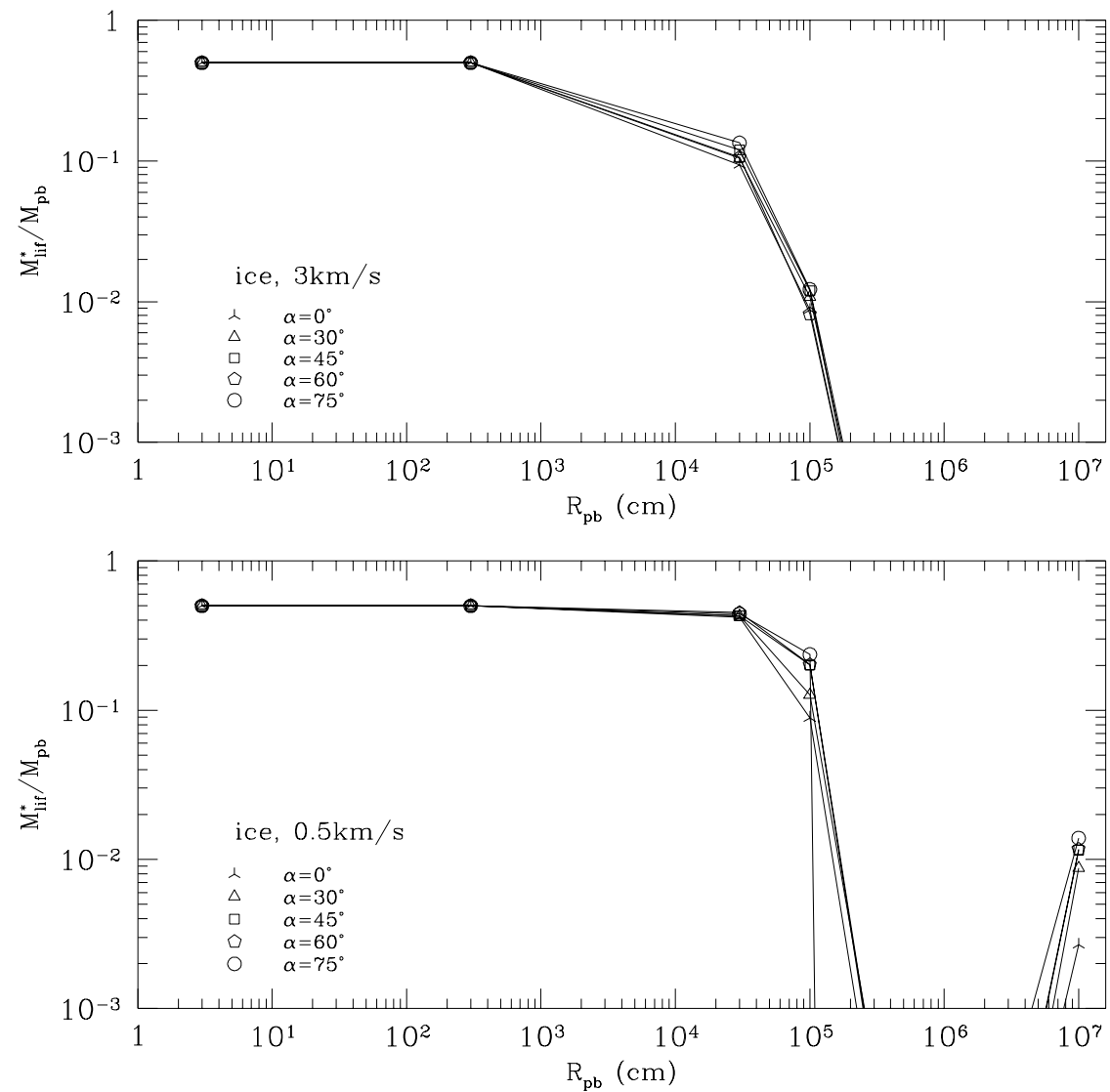

Figure 12: 

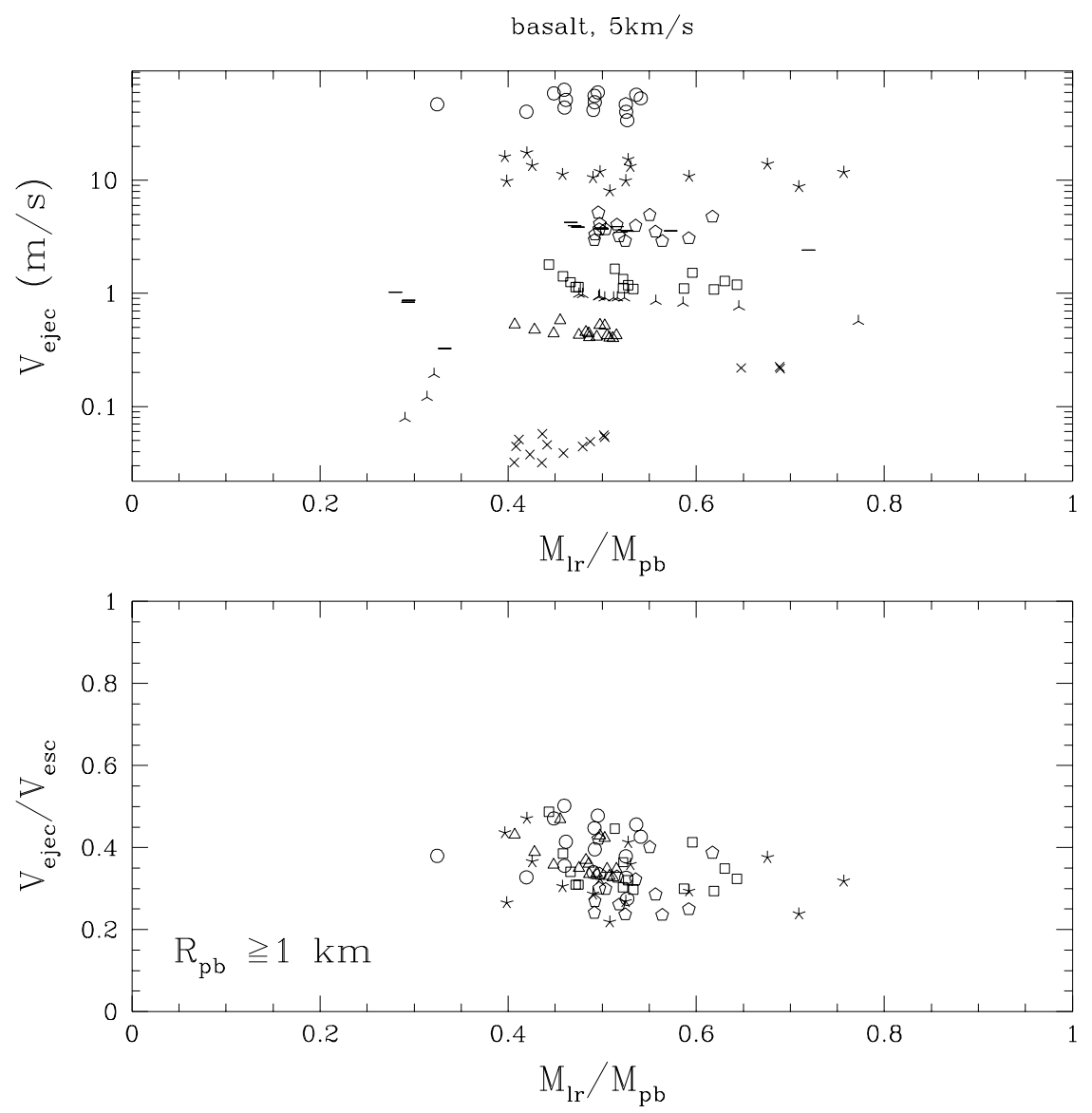

Figure 13: 
basalt, $3 \mathrm{~km} / \mathrm{s}$
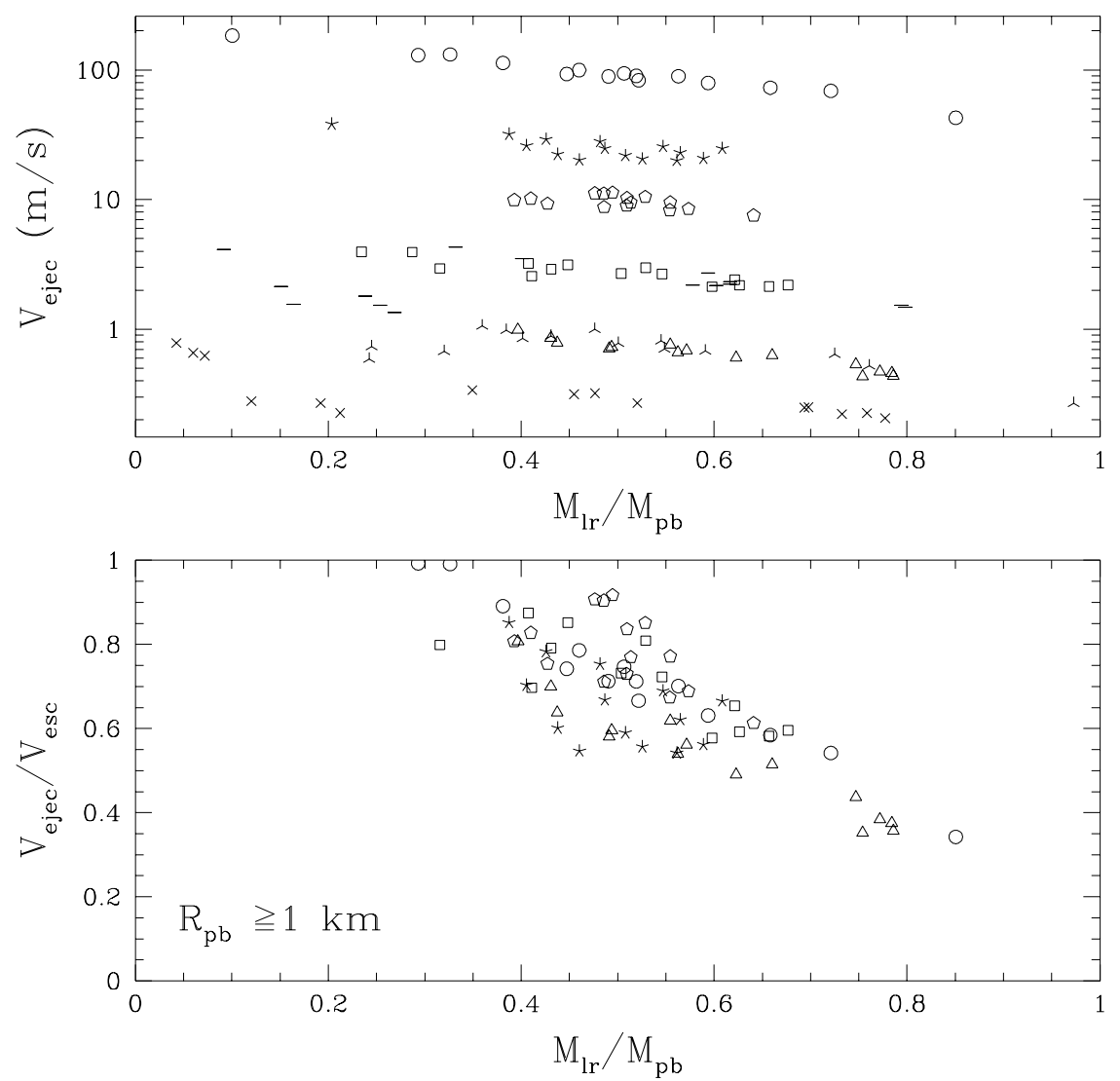

Figure 14: 
ice, $3 \mathrm{~km} / \mathrm{s}$
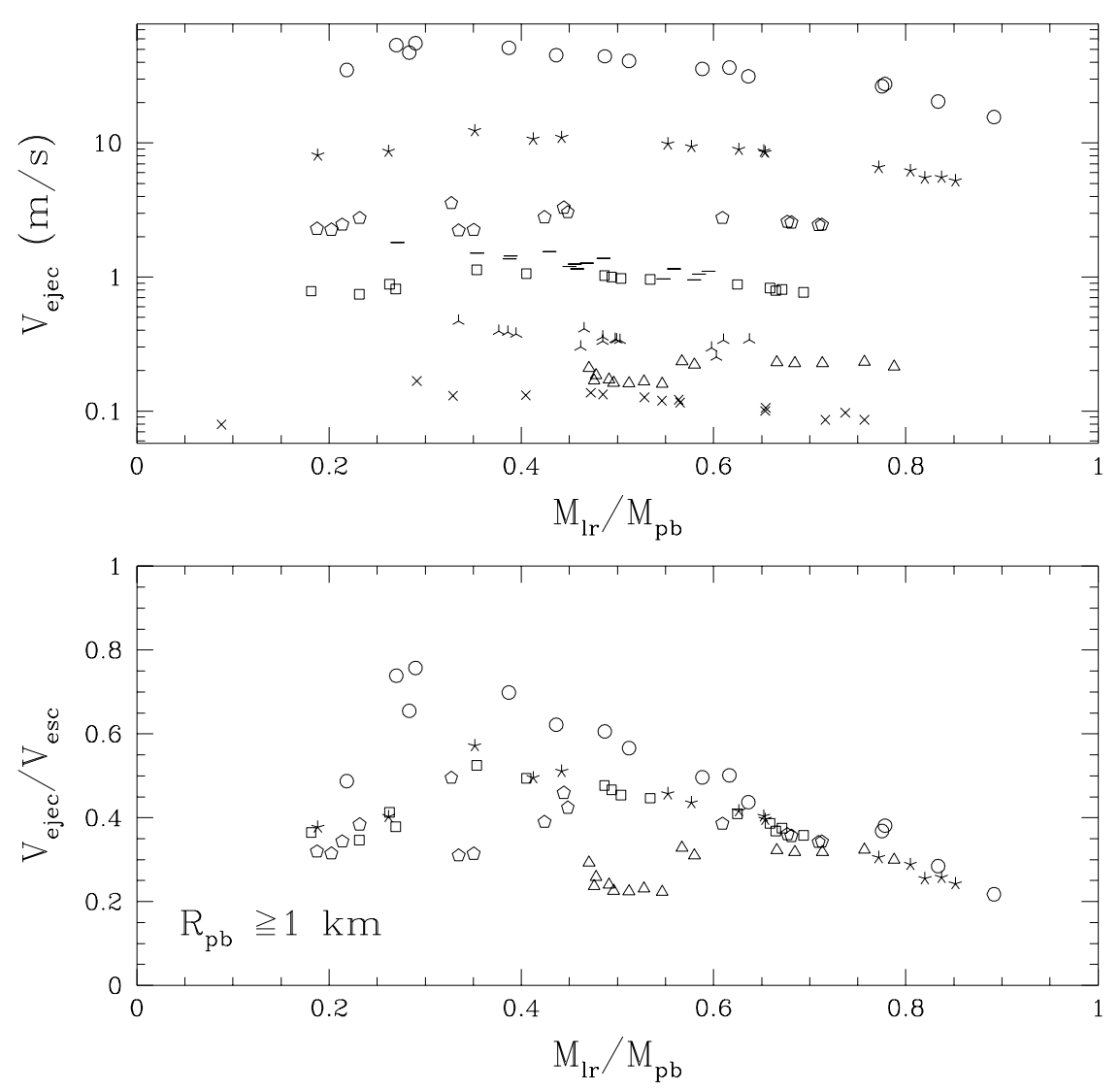

Figure 15: 
ice, $0.5 \mathrm{~km} / \mathrm{s}$
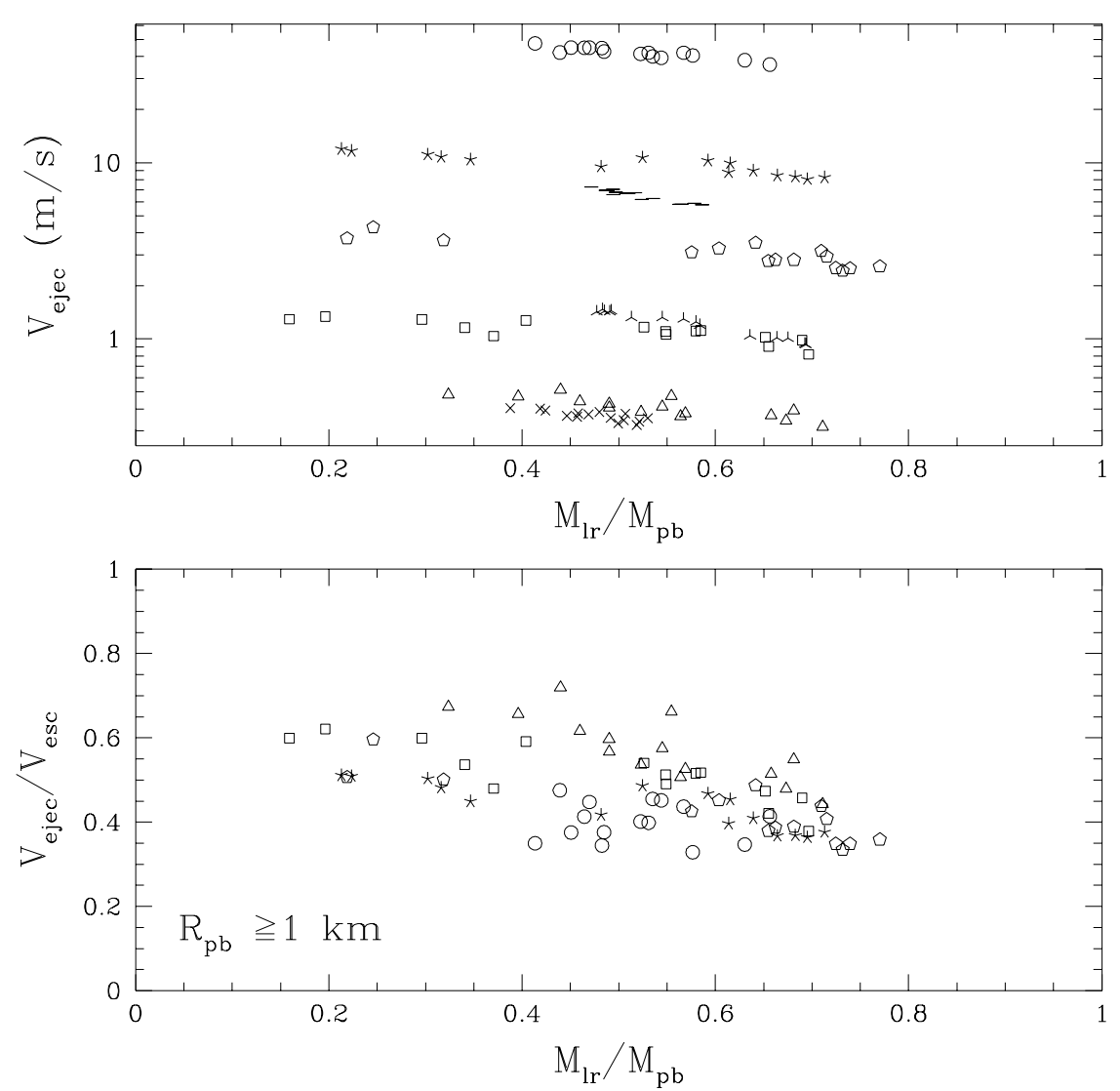

Figure 16: 


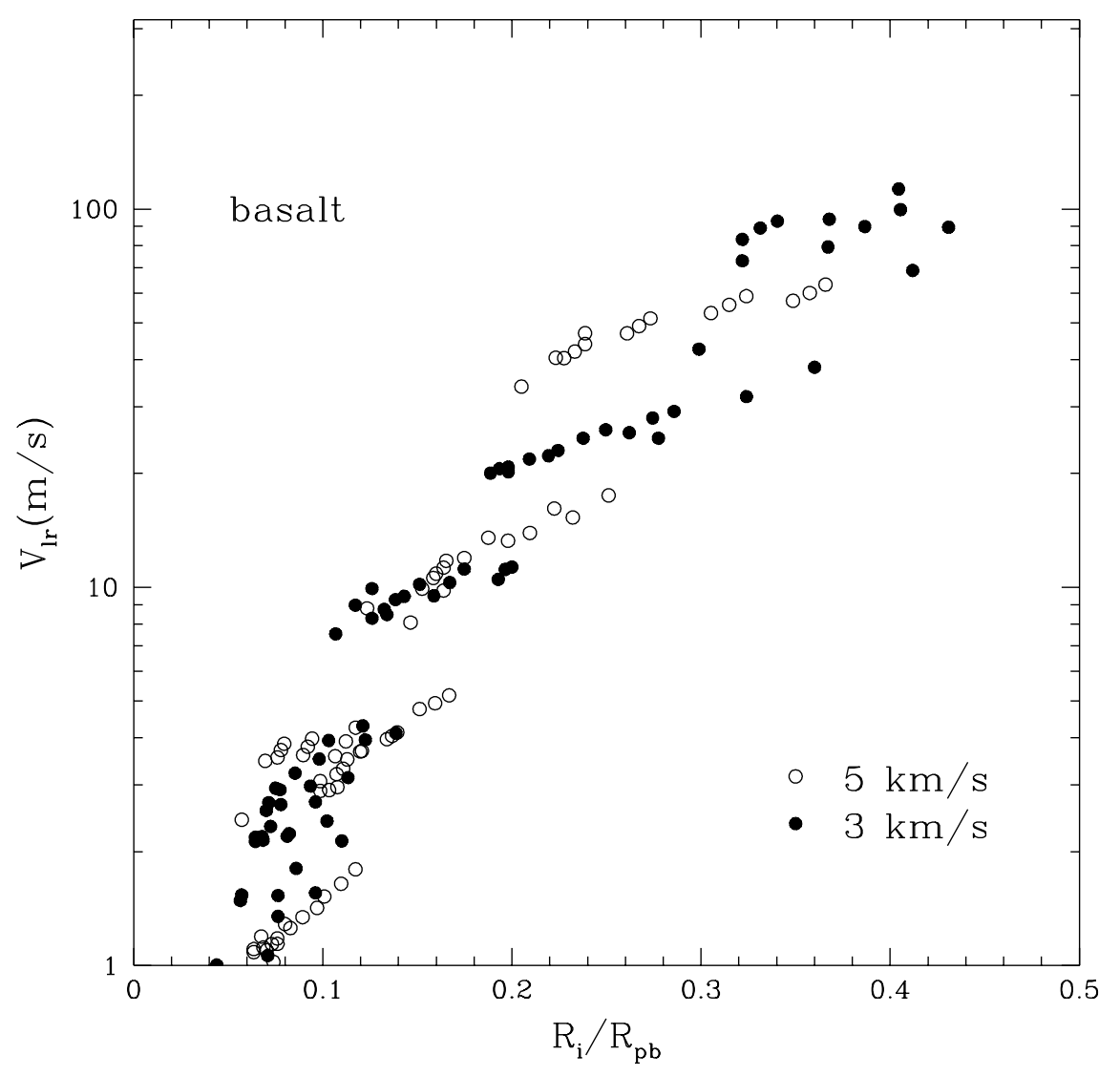

Figure 17: 


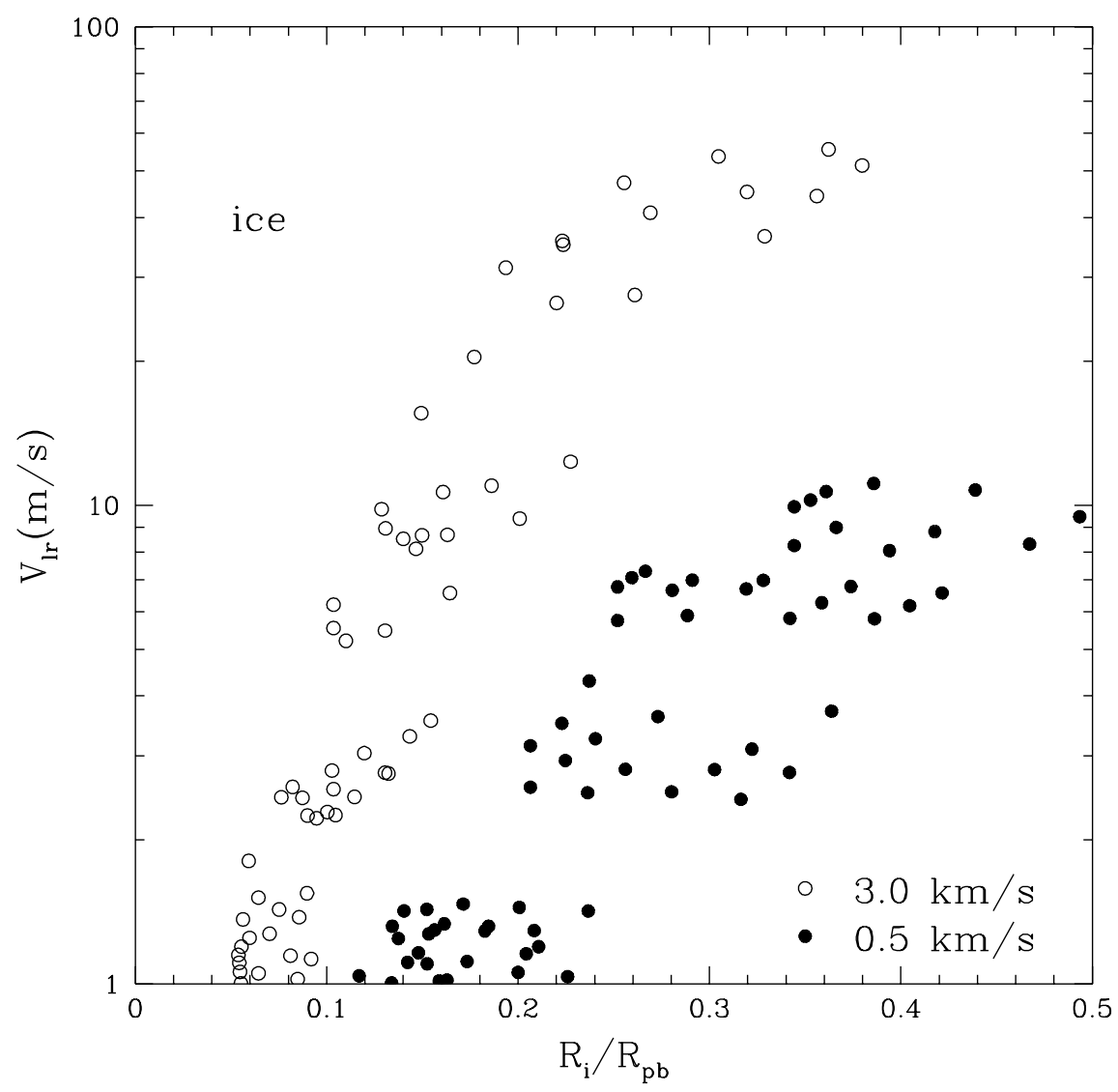

Figure 18: 